Finite-Difference, Time-Domain Simulation of Sound Propagation in a Dynamic Atmosphere

US Army Corps of Engineers ${ }_{\circledast}$ Engineer Research and Development Center

D. Keith Wilson and Lanbo Liu

May 2004 


\section{Finite-Difference, Time-Domain Simulation of Sound Propagation in a Dynamic Atmosphere}

D. Keith Wilson and Lanbo Liu

Approved for public release; distribution is unlimited. 


\section{ABSTRACT}

Finite-difference, time-domain (FDTD) techniques hold much promise for performing realistic simulations of sound propagation through complex, dynamic outdoor environments. This report focuses on a key aspect of FDTD in the atmosphere, namely the incorporation of a moving background medium (wind and turbulence in the atmosphere) into the calculations. Appropriate differential equations for FDTD simulation of sound propagation in a moving fluid are discussed. It is shown that FDTD calculations are not possible with this equation set when using the staggered grid, "leap-frog" approach, which is common for FDTD simulation of other types of wave propagation. Various finite-difference operators that are valid for a moving medium, such as Runge-Kutta and an unstaggered leap-frog approach, are discussed and compared. It is shown that a rigorous FDTD solution in a moving medium requires storing the field variables over at least two time steps, thereby requiring at least twice as much computer memory as the customary staggered grid. Several other topics pertinent to FDTD simulation of sound propagation in the atmosphere are discussed, including implementation of porous ground layers, absorbing boundaries, and rigid surfaces. Example calculations demonstrate the performance of the various finite-difference operators for a high Mach number, uniform flow. Other example calculations show FDTD calculations for propagation above rigid and porous ground surfaces, over rigid barriers, and through turbulence. With sufficiently dense spatial grids, very good agreement can be obtained between the FDTD calculations and known theoretical solutions.

DISCLAIMER: The contents of this report are not to be used for advertising, publication, or promotional purposes. Citation of trade names does not constitute an official endorsement or approval of the use of such commercial products. All product names and trademarks cited are the property of their respective owners. The findings of this report are not to be construed as an official Department of the Army position unless so designated by other authorized documents. 


\section{CONTENTS}

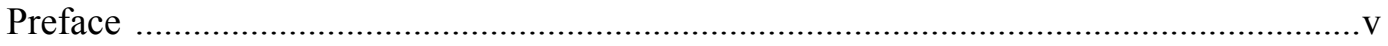

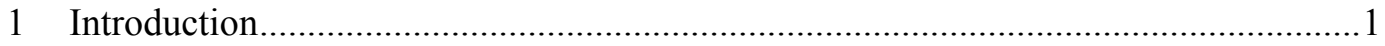

2 Equations for Sound Propagation in a Moving Medium ..........................................

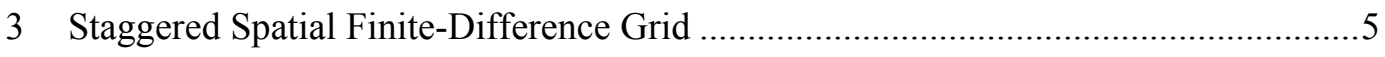

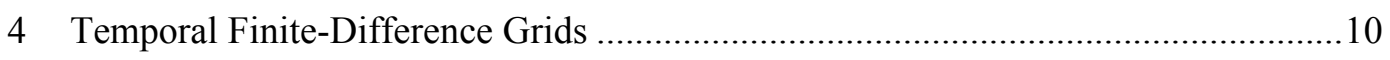

Solution on a Grid Unstaggered in Time ................................................................ 10

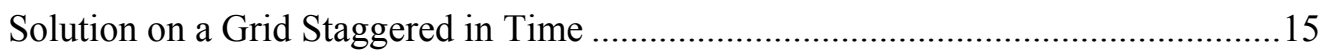

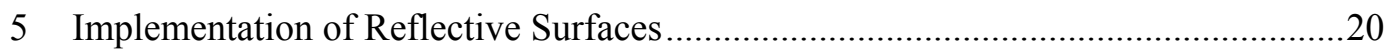

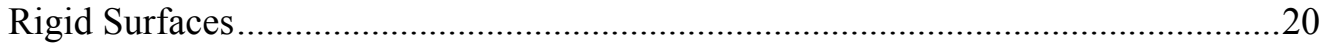

Sound Propagation in a Porous Medium and Implementation ................................2 21

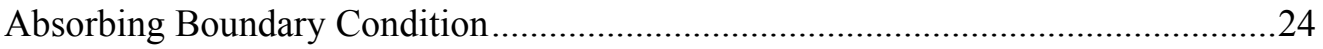

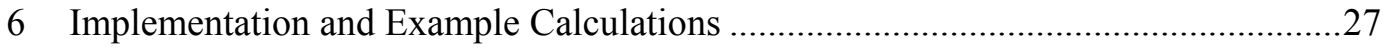

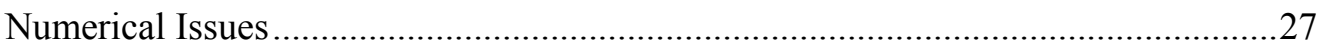

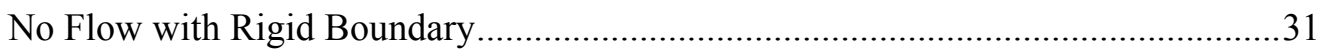

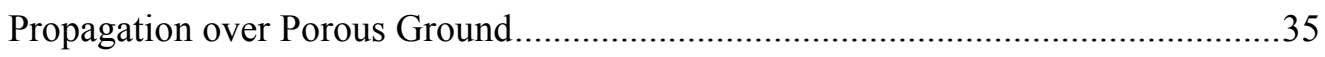

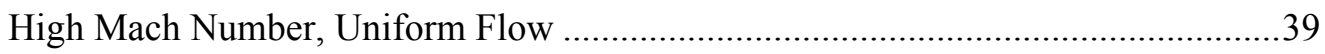

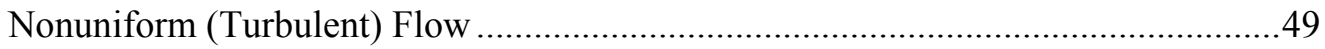

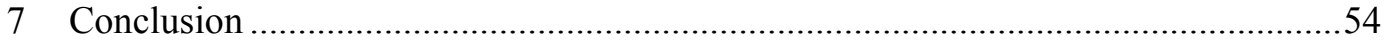

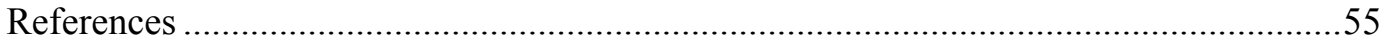

\section{ILLUSTRATIONS}

Figure 1. Spatially staggered finite-difference grid for solution of acoustic wave propagation

Figure 2. Configuration of the computational domain for calculations of the field generated by a harmonic source above a rigid boundary ..................................... 32

Figure 3. Sound pressure field $0.450 \mathrm{~s}$ after initiation of the $100-\mathrm{Hz}$ sound source ....... 33

Figure 4. Comparison of the numerically calculated sound transmission loss with the theoretical result for the low-resolution numerical grid ........................... 34

Figure 5. Comparison of the numerically calculated sound transmission loss with the theoretical result for the high-resolution numerical grid 35 
Figure 6. Configuration of the computational domain for calculations involving porous ground and a rigid barrier

Figure 7. Propagation above soft, porous ground in the presence of a rigid barrier, $0.057 \mathrm{~s}$ after source initiation

Figure 8. Propagation above soft, porous ground in the presence of a rigid barrier, $0.154 \mathrm{~s}$ after source initiation

Figure 9. Propagation above soft, porous ground in the presence of a rigid barrier, $0.249 \mathrm{~s}$ after source initiation 38

Figure 10. Propagation above moderately hard, porous ground in the presence of a rigid barrier, $0.250 \mathrm{~s}$ after source initiation

Figure 11. Sound pressure field in the absence of a background flow, Mach 0 flow ...... 40

Figure 12. Sound pressure field for propagation in a uniform, Mach 0.3 flow ................ 41

Figure 13. Sound pressure field for propagation in a uniform, Mach 0.6 flow ................ 42

Figure 14. Comparison of the angular dependence of the pressure amplitude from the FDTD calculations of Ostashev's theory ................................................ 43

Figure 15. Sound pressure field for propagation in a uniform, Mach 0.3 flow ............... 44

Figure 16. Sound pressure field for propagation in a uniform, Mach 0.3 flow ............... 45

Figure 17. Sound pressure traces for downwind propagation at Mach 0.3 ..................... 46

Figure 18. Sound pressure traces for upwind propagation at Mach 0.3 ........................ 47

Figure 19. Sound pressure traces for downwind propagation at Mach 0.6 .................... 48

Figure 20. Sound pressure traces for upwind propagation at Mach 0.6 .......................... 49

Figure 21. Snapshot of a random turbulence field generated by

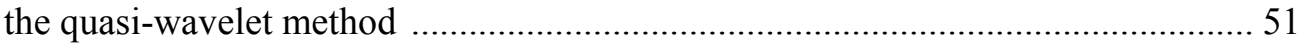

Figure 22. Same as Figure 21, except that the $y$ component of the wind velocity

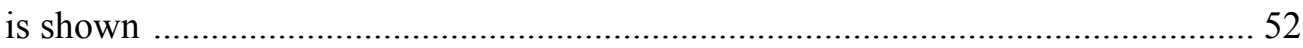

Figure 23. Propagation of a six-cycle burst at $100 \mathrm{~Hz}$ through the turbulence fields shown in Figures 21 and 22

\section{TABLES}

Table 1. Typical values of the static flow resistivity, porosity, and tortuosity for common porous ground surfaces.

Table 2. Comparison of some methods for advancing in time the equations of sound propagation in a moving medium 


\section{PREFACE}

This report was prepared by D. Keith Wilson, Research Physical Scientist, Civil and Infrastructure Engineering Branch, and Lambo Liu, Expert in Geophysics, Snow and Ice Branch, U.S. Army Cold Regions Research and Engineering Laboratory, Engineer Research and Development Center, Hanover, New Hampshire.

The authors are indebted to Vladimir Ostashev of the NOAA Environmental Technology Laboratory, David Aldridge and Neill Symons of the Sandia National Laboratory, David Marlin and Sandra Collier of the Army Research Laboratory, and Steve Ketcham of the U.S. Army Cold Regions Research and Engineering Laboratory for discussions on wave equations in moving media, finite-difference methods, and other topics. Funding for this project was provided by the Program Manager Office for Robotic Unmanned Surveillance, U.S. Army; the DoD High Performance Computing Modernization Office's Common High-Performance Scalable Software Initiative (through the Army Research Laboratory); and the U.S. Army Corps of Engineers, ERDC AT24 direct funding line.

The commander of the Engineer Research and Development Center is COL James R. Rowan, EN. The director is Dr. James R. Houston. 



\title{
Finite-Difference, Time-Domain Simulation of Sound Propagation in a Dynamic Atmosphere
}

\author{
D. KEITH WILSON AND LANBO LIU
}

\section{INTRODUCTION}

Acoustical sensing systems are expected to play a key role in the Army's Future Force by providing non-line-of-sight surveillance over wide areas. It is well known that the performance of battlefield acoustical sensors is affected by complex sound propagation phenomena occurring in outdoor settings, such as reflections from trees and buildings, ground interactions, scattering by turbulence, refraction by wind and temperature gradients, and diffraction over hills. Comprehensive, controlled field studies of the effects of these phenomena on sensors are extremely difficult and expensive to perform. Therefore, a high-quality simulation capable of reproducing these phenomena would be immensely valuable for the development of effective, reliable acoustical sensing systems.

Most currently used numerical methods for outdoor sound propagation, such as the fast field program (FFP) and parabolic equation (PE), are incapable of simulating all of the propagation phenomena mentioned above. [The reader may refer to Attenborough et al. (1995) and Salomons (2001) for detailed discussions of the FFP and PE methods.] On the other hand, many of these phenomena are readily handled with finite-difference, timedomain (FDTD) techniques. Reflections from and diffraction around objects such as buildings, trees, and hills can be readily implemented with the FDTD approach, as can dynamic source inputs and turbulent scattering. But there are significant drawbacks of FDTD that have so far prevented its widespread use in outdoor sound propagation calculations. In particular, it is very computationally intensive. Other inherent difficulties include implementing a time-domain impedance boundary condition for the ground and controlling numerical instabilities at sudden contrasts in material properties. But, given the inherent flexibility of the FDTD technique and the increasing capability of modern computers, FDTD should play an increasingly prominent role in outdoor sound propagation modeling.

Recent papers by Blumrich and Heimann (2002) and Salomons et al. (2002) present 2-D FDTD calculations for the atmosphere that include the effects of motion (wind and 
turbulence) in the propagation medium and address issues related to ground interactions. Liu and Moran (2002) demonstrated 3-D FDTD simulations of propagation in a nonmoving atmosphere with a barrier and a dense stand of trees. Because of the substantial demands on computer memory, the 3-D simulations were run on a distributed, parallelprocessing computer.

In this report we discuss in detail the implementation of FDTD for sound propagation in a moving atmosphere. By "moving," we mean that motion in the medium through which the sound propagates has a significant impact on the propagation, which is generally true when the typical speed of motion in the medium is not very much smaller than the speed of wave propagation through the medium. Motion in the propagation medium has been justifiably neglected in previous FDTD simulations, including seismic propagation in the earth and electromagnetic propagation in the atmosphere, but it cannot be neglected for sound propagation in the atmosphere. For example, the speed of light in air, about $3 \times 10^{8} \mathrm{~m} \mathrm{~s}^{-1}$, is thirty million to one hundred million times larger than typical wind speeds in the atmosphere (3-10 $\mathrm{m} \mathrm{s}^{-1}$ ). The speed of sound, about $340 \mathrm{~m} \mathrm{~s}^{-1}$, is only about 34-110 times larger. As a result, wind and turbulence in the atmosphere have important effects on sound propagation.

In Section 2 of this paper, we discuss the equations for sound propagation in a moving medium. The spatially staggered finite-difference implementation of this equation set, in which the acoustic pressure and particle velocities are stored at different locations in space, is described in Section 3. Section 4 discusses advancement of the solution in time, on grids that are both staggered and unstaggered in time. We point out that the conventional staggered-in-time, "leap-frog" approach for a nonmoving medium cannot be applied directly to a moving medium. The unstaggered-in-time approach allows widely practiced numerical techniques, such as Runge-Kutta integration, to be readily applied to the problem. In Section 5, several other topics pertinent to FDTD simulation of sound propagation in the atmosphere are discussed, including implementation of porous ground layers, absorbing boundaries, and rigid surfaces. Lastly, some example calculations involving propagation through a moving atmosphere are presented in Section 6. 


\section{EQUATIONS FOR SOUND PROPAGATION IN A MOVING MEDIUM}

Most currently used techniques for calculating sound propagation in the atmosphere, such as the fast field program and parabolic equation (FFP and PE), are based on solution of the wave equation or its parabolic approximation. The wave equation is second order with respect to its differential operators in space and time. FDTD, however, is more readily applied to first-order partial differential equations. The following coupled, first-order equations for the acoustic pressure $p$ and acoustic particle velocity w (Ostashev 1987, 1997, Aldridge 2002) serve as an appropriate starting point for FDTD sound propagation calculations in a moving medium:

$$
\begin{aligned}
& \frac{\partial p}{\partial t}=-(\mathbf{v} \cdot \nabla) p-\rho c^{2} \nabla \cdot \mathbf{w}+\rho c^{2} Q, \\
& \frac{\partial \mathbf{w}}{\partial t}=-(\mathbf{w} \cdot \nabla) \mathbf{v}-(\mathbf{v} \cdot \nabla) \mathbf{w}-\frac{\nabla p}{\rho}+\mathbf{F} / \rho .
\end{aligned}
$$

Here, $\rho$ is ambient medium density, $c$ is the adiabatic speed of sound, and $\mathbf{v}$ is the wind velocity. The quantities $\mathbf{F}$ and $Q$ represent sources: the former is a force acting on the medium, whereas the latter is a mass source. (These may be interpreted as dipole and monopole pressure sources, respectively.) As is typical in aeroacoustics, these equations assume that (1) the sound wave is a small perturbation to the background state of the medium, (2) the medium behaves adiabatically (i.e., the material derivative of the specific entropy of the background medium vanishes), and (3) the divergence of the background velocity field (the turbulence) is zero. The gradient of the background pressure is also neglected; this approximation is reasonable for sound propagation near the ground but would need to be reconsidered for infrasound propagating between the ground and upper atmosphere.

The terms $(\mathbf{v} \cdot \nabla) p$ and $(\mathbf{v} \cdot \nabla) \mathbf{w}$ in Eq. 1 and 2 represent the transport of the sound wave by the atmospheric wind. These are commonly called advective terms. The term $(\mathbf{w} \cdot \nabla) \mathbf{v}$ in Eq. 2 represents enhancement/diminishment of the acoustic particle velocity by spatial variations in the wind field. For a nonmoving medium $(\mathbf{v}=0)$, one can derive the customary wave equation for the acoustic pressure by taking the time derivative of Eq. 1 and the divergence of Eq. 2. However, the additional terms involving the wind velocity introduce significant complications when attempting to derive wave equations or parabolic equations for use in an FFP or PE (Pierce 1990, Ostashev et al. 1997, Lingevitch et al. 2002). An FDTD code based on the first-order Eq. 1 and 2 would be 
more general and accurate than most current sound propagation formulations, despite the fairly simple appearance of the differential equations.

In this report we consider the FD implementation of Eq. 1 and 2 in two dimensions. This simplifies the presentation considerably and makes it possible to run calculations on single-processor computers. The generalization of the results to three dimensions is quite straightforward but more computationally intensive. In two dimensions, Eq. 1 and 2 become

$$
\begin{aligned}
& \frac{\partial p}{\partial t}=-\left(v_{x} \frac{\partial}{\partial x}+v_{y} \frac{\partial}{\partial y}\right) p-\kappa\left(\frac{\partial w_{x}}{\partial x}+\frac{\partial w_{y}}{\partial y}\right)+\kappa Q, \\
& \frac{\partial w_{x}}{\partial t}=-\left(w_{x} \frac{\partial}{\partial x}+w_{y} \frac{\partial}{\partial y}\right) v_{x}-\left(v_{x} \frac{\partial}{\partial x}+v_{y} \frac{\partial}{\partial y}\right) w_{x}-b \frac{\partial p}{\partial x}+b F_{x},
\end{aligned}
$$

and

$$
\frac{\partial w_{y}}{\partial t}=-\left(w_{x} \frac{\partial}{\partial x}+w_{y} \frac{\partial}{\partial y}\right) v_{y}-\left(v_{x} \frac{\partial}{\partial x}+v_{y} \frac{\partial}{\partial y}\right) w_{y}-b \frac{\partial p}{\partial y}+b F_{y}
$$

where $b=1 / \rho$ is the mass buoyancy and $c=\rho c^{2}$ is the adiabatic bulk modulus. 


\section{STAGGERED SPATIAL FINITE-DIFFERENCE GRID}

In this section we consider second-order approximations of the spatial derivatives in Eq. 3-5. The spatial finite-difference analysis in this report is based entirely on a grid for the pressure and particle velocities that is staggered in space, as shown in Figure 1. In this grid the pressure is stored at whole nodes, namely $x=i \Delta x$ and $y=j \Delta y$, where $i$ and $j$ are integers and $\Delta x$ and $\Delta y$ are the grid spacings in the $x$ and $y$ directions." The $x$ components of the acoustic particle velocity, $w_{x}$, are staggered (offset) by $\Delta x / 2$ in the $x$ direction. The $y$ components of the acoustic particle velocity, $w_{y}$, are staggered by $\Delta y / 2$ in the $y$ direction. An additional convention in this report is that the quantities $b, \kappa$, and $Q$ are stored at the pressure nodes. The quantities $v_{x}$ and $F_{x}$ are stored at the $w_{x}$ nodes, and $v_{y}$ and $F_{y}$ are stored at the $w_{y}$ nodes.

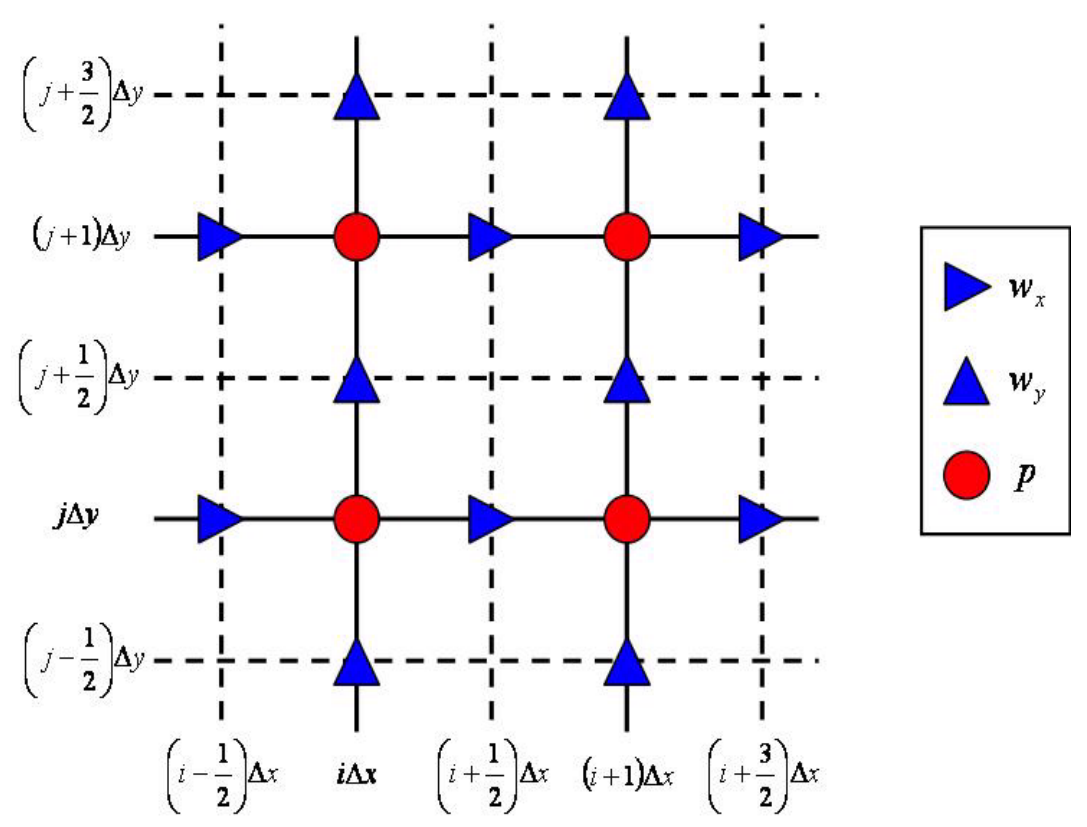

Figure 1. Spatially staggered finite-difference grid for solution of acoustic wave propagation.

Centered, finite-difference solution of Eq. 3-5 requires an evaluation of the righthand sides of these equations at the grid nodes where the field variable is stored. For

\footnotetext{
*The spatially staggered grid in Fig. 1 matches the one normally used for wave propagation calculations in nonmoving media. Given the difficulties of applying the staggered grid to a moving medium, it would be worthwhile considering the potential advantages of spatially unstaggered grids in this context. Such consideration is not given in this report, however.
} 
example, we will need finite-difference approximations, centered at $x=i \Delta x$ and $y=j \Delta y$, for all terms on the right-hand side of Eq. 3. Finite difference approximations centered on $x=(i+1 / 2) \Delta x$ and $y=j \Delta y$ are needed for Eq. 4, whereas approximations centered on $x=$ $i \Delta x$ and $y=(j+1 / 2) \Delta y$ are needed for Eq. 5 .

Consider first the source terms and coefficients involving the medium properties. The source terms can be used directly, since they are already stored at the grid nodes where the FD approximations are centered. The same is true of $\kappa$, which is stored at the pressure grid nodes and needed in Eq. 3. Regarding Eq. 4 and 5, the values for $b$ can be determined at the needed locations by averaging neighboring values:

$$
\begin{aligned}
& b[(i+1 / 2) \Delta x, j \Delta y, t] \simeq \frac{b[(i+1) \Delta x, j \Delta y, t]+b[i \Delta x, j \Delta y, t]}{2}, \\
& b[i \Delta x,(j+1 / 2) \Delta y, t] \simeq \frac{b[i \Delta x,(j+1) \Delta y, t]+b[i \Delta x, j \Delta y, t]}{2} .
\end{aligned}
$$

One of the main motivations for using the spatially staggered grid is that it conveniently provides centered spatial differences for many of the derivatives in Eq. 3-5. In particular,

$$
\begin{aligned}
& \frac{\partial w_{x}(i \Delta x, j \Delta y, t)}{\partial x} \simeq \frac{w_{x}[(i+1 / 2) \Delta x, j \Delta y, t]-w_{x}[(i-1 / 2) \Delta x, j \Delta y, t]}{\Delta x}, \\
& \frac{\partial w_{y}(i \Delta x, j \Delta y, t)}{\partial y} \simeq \frac{w_{y}[i \Delta x,(j+1 / 2) \Delta y, t]-w_{y}[i \Delta x,(j-1 / 2) \Delta y, t]}{\Delta y}, \\
& \frac{\partial p[(i+1 / 2) \Delta x, j \Delta y, t]}{\partial x} \simeq \frac{p[(i+1) \Delta x, j \Delta y, t]-p[i \Delta x, j \Delta y, t]}{\Delta x}
\end{aligned}
$$

and

$$
\frac{\partial p[i \Delta x,(j+1 / 2) \Delta y, t]}{\partial y} \simeq \frac{p[i \Delta x,(j+1) \Delta y, t]-p[i \Delta x, j \Delta y, t]}{\Delta y}
$$

At this point we have developed centered approximations for all of the terms in Eq. $3-5$ that are present for a nonmoving medium. Implementation of the remaining terms is 
somewhat more complicated, however. For example, the derivatives of the pressure field in Eq. 3, $\partial p / \partial x$ and $\partial p / \partial y$, cannot be centered at $x=i \Delta x$ and $y=j \Delta y$ from approximations across a single grid interval. We can develop centered approximations across two grid intervals, however:

$$
\frac{\partial p(i \Delta x, j \Delta y, t)}{\partial x} \simeq \frac{p[(i+1) \Delta x, j \Delta y, t]-p[(i-1) \Delta x, j \Delta y, t]}{2 \Delta x}
$$

and

$$
\frac{\partial p(i \Delta x, j \Delta y, t)}{\partial y} \simeq \frac{p[i \Delta x,(j+1) \Delta y, t]-p[i \Delta x,(j-1) \Delta y, t]}{2 \Delta y}
$$

To find the wind velocity components $v_{x}$ and $v_{y}$ evaluated at $x=i \Delta x$ and $y=j \Delta y$ (multiplying the derivatives $\partial p / \partial x$ and $\partial p / \partial y$, respectively, in Eq. 3), we average values at neighboring grid points:

$$
\begin{aligned}
& v_{x}(i \Delta x, j \Delta y, t) \simeq \frac{v_{x}[(i+1 / 2) \Delta x, j \Delta y, t]+v_{x}[(i-1 / 2) \Delta x, j \Delta y, t]}{2}, \\
& v_{y}(i \Delta x, j \Delta y, t) \simeq \frac{v_{y}[\Delta x,(j+1 / 2) \Delta y, t]+v_{y}[i \Delta x,(j-1 / 2) \Delta y, t]}{2} .
\end{aligned}
$$

A similar approach to Eq. 4 and 5 leads to the approximations

$$
\begin{aligned}
& \frac{\partial w_{x}[(i+1 / 2) \Delta x, j \Delta y, t]}{\partial x} \\
& \simeq \frac{w_{x}[(i+3 / 2) \Delta x, j \Delta y, t]-w_{x}[(i-1 / 2) \Delta x, j \Delta y, t]}{2 \Delta x}, \\
& \frac{\partial w_{x}[(i+1 / 2) \Delta x, j \Delta y, t]}{\partial y} \\
& \simeq \frac{w_{x}[(i+1 / 2) \Delta x,(j+1) \Delta y, t]-w_{x}[(i+1 / 2) \Delta x,(j-1) \Delta y, t]}{2 \Delta y},
\end{aligned}
$$




$$
\begin{aligned}
& \frac{\partial w_{y}[i \Delta x,(j+1 / 2) \Delta y, t]}{\partial x} \\
& \quad \simeq \frac{w_{y}[(i+1) \Delta x,(j+1 / 2) \Delta y, t]-w_{y}[(i-1) \Delta x,(j+1 / 2) \Delta y, t]}{2 \Delta x},
\end{aligned}
$$

and

$$
\begin{aligned}
& \frac{\partial w_{y}[i \Delta x,(j+1 / 2) \Delta y, t]}{\partial y} \\
& \simeq \frac{w_{y}[i \Delta x,(j+3 / 2) \Delta y, t]-w_{y}[i \Delta x,(j-1 / 2) \Delta y, t]}{2 \Delta y} .
\end{aligned}
$$

The same approximations hold for derivatives of the wind field when $w_{x}$ is replaced by $v_{x}$ and $w_{y}$ by $v_{y}$. In Eq. 4 the quantities $w_{y}$ and $v_{y}$ (multiplying the derivatives $\partial v_{x} / \partial y$ and $\partial w_{x} / \partial y$, respectively) are needed at the grid point $x=(i+1 / 2) \Delta x$ and $y=j \Delta y$. Referring to Figure 1, a reasonable way to obtain these quantities would be to average the four closest grid nodes:

$$
\begin{aligned}
& w_{y}[(i+1 / 2) \Delta x, j \Delta y, t] \\
& \simeq \frac{1}{4}\left\{w_{y}[(i+1) \Delta x,(j+1 / 2) \Delta y, t]+w_{y}[i \Delta x,(j+1 / 2) \Delta y, t]\right. \\
& \left.\quad+w_{y}[(i+1) \Delta x,(j-1 / 2) \Delta y, t]+w_{y}[i \Delta x,(j-1 / 2) \Delta y, t]\right\},
\end{aligned}
$$

and likewise for $v_{y}$. Similarly, in Eq. 5 the quantities $w_{x}$ and $v_{x}$ (multiplying the derivatives $\partial v_{y} / \partial x$ and $\partial w_{y} / \partial x$, respectively) are needed at the grid point $x=i \Delta x$ and $y=(j+1 / 2) \Delta y$. We have

$$
\begin{aligned}
& w_{x}[i \Delta x,(j+1 / 2) \Delta y, t] \\
& \simeq \frac{1}{4}\left\{w_{x}[(i+1 / 2) \Delta x,(j+1) \Delta y, t]+w_{x}[(i+1 / 2) \Delta x, j \Delta y, t]\right. \\
& \left.\quad+w_{x}[(i-1 / 2) \Delta x,(j+1) \Delta y, t]+w_{x}[(i-1 / 2) \Delta x, j \Delta y, t]\right\},
\end{aligned}
$$

and likewise for $v_{x}$. 
Equations 6-21 provide finite-difference approximations for all of the quantities appearing on the right-hand sides of Eq. 3-5. We next turn our attention to finitedifference approximations for the temporal derivatives. 


\section{TEMPORAL FINITE-DIFFERENCE GRIDS}

In this section we consider two alternative approaches to advancing the wavefield variables in time. The first is based on an unstaggered-in-time grid, meaning that the pressure and acoustic particle velocities are stored on the same time level. The second is based on a staggered-in-time grid, in which the pressure and particle velocities are stored on alternating half time steps.

\section{Solution on a Grid Unstaggered in Time}

Let us define $f_{p}$ as the right-hand side of Eq. 3. We write

$$
\frac{\partial p(i \Delta x, j \Delta y, t)}{\partial t}=f_{p}\left[i \Delta x, j \Delta y, \mathbf{p}(t), \mathbf{w}_{x}(t), \mathbf{w}_{y}(t), \mathbf{s}(t)\right],
$$

where $\mathbf{p}(t), \mathbf{w}_{x}(t)$, and $\mathbf{w}_{y}(t)$ represent matrices containing the pressures and particle velocities at all available grid nodes. Furthermore, $\mathbf{s}(t)$ represents the source and medium properties $\left(b, \kappa, v_{x}, v_{y}, Q, F_{x}\right.$, and $\left.F_{y}\right)$ at all available grid nodes. ${ }^{*}$ From Eq. 8-9 and 1215 , the second-order FD approximation of $f_{p}$ is

$$
\begin{aligned}
f_{p}\left[i \Delta x, j \Delta y, \mathbf{p}(t), \mathbf{w}_{x}(t), \mathbf{w}_{y}(t), \mathbf{s}(t)\right] \simeq \\
-\frac{1}{4 \Delta x}\left\{v_{x}[(i+1 / 2) \Delta x, j \Delta y, t]+v_{x}[(i-1 / 2) \Delta x, j \Delta y, t]\right\} \\
\quad \times\{p[(i+1) \Delta x, j \Delta y, t]-p[(i-1) \Delta x, j \Delta y, t]\} \\
-\frac{1}{4 \Delta y}\left\{v_{y}[\Delta x,(j+1 / 2) \Delta y, t]+v_{y}[i \Delta x,(j-1 / 2) \Delta y, t]\right\} \\
\quad \times\{p[i \Delta x,(j+1) \Delta y, t]-p[i \Delta x,(j-1) \Delta y, t]\} \\
-\kappa(i \Delta x, j \Delta y, t)\left\{\frac{w_{x}[(i+1 / 2) \Delta x, j \Delta y, t]-w_{x}[(i-1 / 2) \Delta x, j \Delta y, t]}{\Delta x}\right. \\
\left.+\frac{w_{y}[i \Delta x,(j+1 / 2) \Delta y, t]-w_{y}[i \Delta x,(j-1 / 2) \Delta y, t]}{\Delta y}\right\} \\
+\kappa(i \Delta x, j \Delta y, t) Q(i \Delta x, j \Delta y, t) .
\end{aligned}
$$

*Note that $f_{p}\left[i \Delta x, j \Delta y, \mathbf{p}(t), \mathbf{w}_{x}(t), \mathbf{w}_{y}(t), \mathbf{s}(t)\right]$ in actuality depends only on the fields at grid points in the immediate vicinity of $(i \Delta x, j \Delta y)$ when second-order spatial differencing is used. The notation used here, which is convenient for discussion purposes, is general enough to accommodate spatial differencing of an arbitrarily high order as well as staggered and unstaggered spatial grids. 
Similarly to Eq. 22 we define $f_{x}$ and $f_{y}$ as the right-hand sides of Eq. 4 and 5:

$$
\frac{\partial w_{x}[(i+1 / 2) \Delta x, j \Delta y, t]}{\partial t}=f_{x}\left[(i+1 / 2) \Delta x, j \Delta y, \mathbf{p}(t), \mathbf{w}_{x}(t), \mathbf{w}_{y}(t), \mathbf{s}(t)\right]
$$

and

$$
\frac{\partial w_{y}[i \Delta x,(j+1 / 2) \Delta y, t]}{\partial t}=f_{y}\left[i \Delta x,(j+1 / 2) \Delta y, \mathbf{p}(t), \mathbf{w}_{x}(t), \mathbf{w}_{y}(t), \mathbf{s}(t)\right]
$$

where, from the equations in Section 3,

$$
\begin{aligned}
& f_{x}\left[(i+1 / 2) \Delta x, j \Delta y, \mathbf{p}(t), \mathbf{w}_{x}(t), \mathbf{w}_{y}(t), \mathbf{s}(t)\right] \simeq \\
& -\frac{w_{x}[(i+1 / 2) \Delta x, j \Delta y, t]}{2 \Delta x}\left\{v_{x}[(i+3 / 2) \Delta x, j \Delta y, t]-v_{x}[(i-1 / 2) \Delta x, j \Delta y, t]\right\} \\
& -\frac{1}{8 \Delta y}\left\{w_{y}[(i+1) \Delta x,(j+1 / 2) \Delta y, t]+w_{y}[i \Delta x,(j+1 / 2) \Delta y, t]\right. \\
& \left.+w_{y}[(i+1) \Delta x,(j-1 / 2) \Delta y, t]+w_{y}[i \Delta x,(j-1 / 2) \Delta y, t]\right\} \\
& \quad \times\left\{v_{x}[(i+1 / 2) \Delta x,(j+1) \Delta y, t]-v_{x}[(i+1 / 2) \Delta x,(j-1) \Delta y, t]\right\} \\
& -\frac{v_{x}[(i+1 / 2) \Delta x, j \Delta y, t]}{2 \Delta x}\left\{w_{x}[(i+3 / 2) \Delta x, j \Delta y, t]-w_{x}[(i-1 / 2) \Delta x, j \Delta y, t]\right\} \\
& -\frac{1}{8 \Delta y}\left\{v_{y}[(i+1) \Delta x,(j+1 / 2) \Delta y, t]+v_{y}[i \Delta x,(j+1 / 2) \Delta y, t]\right. \\
& \left.+v_{y}[(i+1) \Delta x,(j-1 / 2) \Delta y, t]+v_{y}[i \Delta x,(j-1 / 2) \Delta y, t]\right\} \\
& \times\left\{w_{x}[(i+1 / 2) \Delta x,(j+1) \Delta y, t]-w_{x}[(i+1 / 2) \Delta x,(j-1) \Delta y, t]\right\} \\
& +\frac{1}{2 \Delta x}\{b[(i+1) \Delta x, j \Delta y, t]+b[i \Delta x, j \Delta y, t]\} \\
& \times\{p[(i+1) \Delta x, j \Delta y, t]-p[i \Delta x, j \Delta y, t]\} \\
& 2(i+1) \Delta x, j \Delta y, t]+b[i \Delta x, j \Delta y, t] F_{x}[(i+1 / 2) \Delta x, j \Delta y, t] \\
& 2
\end{aligned}
$$

and 


$$
\begin{aligned}
& f_{y}\left[i \Delta x,(j+1 / 2) \Delta y, \mathbf{p}(t), \mathbf{w}_{x}(t), \mathbf{w}_{y}(t), \mathbf{s}(t)\right] \simeq \\
& -\frac{1}{8 \Delta x}\left\{w_{y}[(i+1 / 2) \Delta x,(j+1) \Delta y, t]+w_{x}[(i+1 / 2) \Delta x, j \Delta y, t]\right. \\
& \left.+w_{x}[(i-1 / 2) \Delta x,(j+1) \Delta y, t]+w_{x}[(i-1 / 2) \Delta x, j \Delta y, t]\right\} \\
& \times\left\{v_{y}[(i+1) \Delta x,(j+1 / 2) \Delta y, t]-v_{y}[(i-1) \Delta x,(j+1 / 2) \Delta y, t]\right\} \\
& -\frac{w_{y}[i \Delta x,(j+1 / 2) \Delta y, t]}{2 \Delta y}\left\{v_{y}[i \Delta x,(j+3 / 2) \Delta y, t]-v_{y}[i \Delta x,(j-1 / 2) \Delta y, t]\right\} \\
& -\frac{1}{8 \Delta x}\left\{v_{x}[(i+1 / 2) \Delta x,(j+1) \Delta y, t]+v_{x}[(i+1 / 2) \Delta x, j \Delta y, t]\right. \\
& \left.+v_{x}[(i-1 / 2) \Delta x,(j+1) \Delta y, t]+v_{x}[(i-1 / 2) \Delta x, j \Delta y, t]\right\} \\
& \times\left\{w_{y}[(i+1) \Delta x,(j+1 / 2) \Delta y, t]-w_{y}[(i-1) \Delta x,(j+1 / 2) \Delta y, t]\right\} \\
& -\frac{v_{y}[i \Delta x,(j+1 / 2) \Delta y, t]}{2 \Delta y}\left\{w_{y}[i \Delta x,(j+3 / 2) \Delta y, t]-w_{y}[i \Delta x,(j-1 / 2) \Delta y, t]\right\} \\
& -\frac{1}{2 \Delta y}\{b[i \Delta x,(j+1) \Delta y, t]+b[i \Delta x, j \Delta y, t]\} \\
& \times\{p[i \Delta x,(j+1) \Delta y, t]-p[i \Delta x, j \Delta y, t]\} \\
& +\frac{b[i \Delta x,(j+1) \Delta y, t]+b[i \Delta x, j \Delta y, t]}{2} F_{y}[i \Delta x,(j+1 / 2) \Delta y, t] .
\end{aligned}
$$

Suppose now that $\mathbf{p}, \mathbf{w}_{x}, \mathbf{w}_{y}$, and $\mathbf{s}$ are stored at discrete time levels $t=l \Delta t$. A simple approximation to the time derivatives that we could consider is the forward-difference approximation, namely,

$$
\frac{\partial p(i \Delta x, j \Delta y, l \Delta t)}{\partial t} \simeq \frac{p[i \Delta x, j \Delta y,(l+1) \Delta t]-p[i \Delta x, j \Delta y, l \Delta t]}{\Delta t}
$$

and similarly for $\partial w_{x}[(i+1 / 2) \Delta x, j \Delta y, l \Delta t] / \partial t$ and $\partial w_{y}[i \Delta x,(j+1 / 2) \Delta y, l \Delta t] / \partial t$. Solving for the variables at the time $t=(l+1) \Delta t$ gives 


$$
\begin{gathered}
p[i \Delta x, j \Delta y,(l+1) \Delta t]=p[i \Delta x, j \Delta y, l \Delta t] \\
+\Delta t f_{p}\left[i \Delta x, j \Delta y, \mathbf{p}(l \Delta t), \mathbf{w}_{x}(l \Delta t), \mathbf{w}_{y}(l \Delta t), \mathbf{s}(l \Delta t)\right], \\
w_{x}[(i+1 / 2) \Delta x, j \Delta y,(l+1) \Delta t]=w_{x}[(i+1 / 2) \Delta x, j \Delta y, l \Delta t] \\
\quad+\Delta t f_{x}\left[(i+1 / 2) \Delta x, j \Delta y, \mathbf{p}(l \Delta t), \mathbf{w}_{x}(l \Delta t), \mathbf{w}_{y}(l \Delta t), \mathbf{s}(l \Delta t)\right],
\end{gathered}
$$

and

$$
\begin{aligned}
& w_{y} {[i \Delta x,(j+1 / 2) \Delta y,(l+1) \Delta t]=w_{y}[i \Delta x,(j+1 / 2) \Delta y, l \Delta t] } \\
&+\Delta t f_{y}\left[i \Delta x,(j+1 / 2) \Delta y, \mathbf{p}(l \Delta t), \mathbf{w}_{x}(l \Delta t), \mathbf{w}_{y}(l \Delta t), \mathbf{s}(l \Delta t)\right] .
\end{aligned}
$$

This method of advancing the variables in time based on forward differences is often called Euler's method (e.g., Burden et al. 1981, Kreyszig 1988). Unfortunately, as discussed in Kasahara (1977), this method is unconditionally unstable. Even when the time step is made very small, the error will grow exponentially and eventually become unacceptable.

A better approach is to center the approximations for the time derivatives by using fields from two previous time steps:

$$
\frac{\partial p(i \Delta x, j \Delta y, l \Delta t)}{\partial t} \simeq \frac{p[i \Delta x, j \Delta y,(l+1) \Delta t]-p[i \Delta x, j \Delta y,(l-1) \Delta t]}{2 \Delta t} .
$$

Note that the derivative at $t=l \Delta t$ is being estimated from the fields at $t=(l-1) \Delta t$ and $t=(l+1) \Delta t$. This leads to the updating equation

$$
\begin{aligned}
& p[i \Delta x, j \Delta y,(l+1) \Delta t]=p[i \Delta x, j \Delta y,(l-1) \Delta t] \\
& \quad+2 \Delta t f_{p}\left[i \Delta x, j \Delta y, \mathbf{p}(l \Delta t), \mathbf{w}_{x}(l \Delta t), \mathbf{w}_{y}(l \Delta t), \mathbf{s}(l \Delta t)\right],
\end{aligned}
$$

and similarly for $w_{x}$ and $w_{y}$. Kasahara (1977) calls this method the "leap-frog" scheme. Unlike the Euler scheme, it is generally stable. The leap-frog scheme was one of the most common in atmospheric modeling at the time of Kasahara's writing. As will be shown later with examples, it is also a satisfactory scheme for calculating sound propagation in a moving medium. However, the field variables must be stored over two time steps, thereby doubling memory requirements. In recent decades the leap-frog scheme has been 
replaced by somewhat more complicated ones that usually provide more accurate calculations with little additional computational burden.

One important class of numerical methods that is in widespread usage today is the Runge-Kutta methods (Burden et al. 1981, Kreyszig 1988). The second-order RungeKutta method (also called Heun's method or the Improved Euler method) can be motivated in an intuitive, heuristic manner. It begins in the same way as Euler's method, by forming an estimate for the field at the next time step based on Eq. 29-31. These approximations are indicated here with superscripted asterisks, i.e.,

$$
\begin{aligned}
& p^{*}[i \Delta x, j \Delta y,(l+1) \Delta t]=p[i \Delta x, j \Delta y, l \Delta t] \\
& +\Delta t f_{p}\left[i \Delta x, j \Delta y, \mathbf{p}(l \Delta t), \mathbf{w}_{x}(l \Delta t), \mathbf{w}_{y}(l \Delta t), \mathbf{s}(l \Delta t)\right], \\
& w_{x}^{*}[(i+1 / 2) \Delta x, j \Delta y,(l+1) \Delta t]=w_{x}[(i+1 / 2) \Delta x, j \Delta y, l \Delta t] \\
& \quad+\Delta t f_{x}\left[(i+1 / 2) \Delta x, j \Delta y, \mathbf{p}(l \Delta t), \mathbf{w}_{x}(l \Delta t), \mathbf{w}_{y}(l \Delta t), \mathbf{s}(l \Delta t)\right], \\
& w_{y}^{*}[i \Delta x,(j+1 / 2) \Delta y,(l+1) \Delta t]=w_{y}[i \Delta x,(j+1 / 2) \Delta y, l \Delta t] \\
& \quad+t f_{y}\left[i \Delta x,(j+1 / 2) \Delta y, \mathbf{p}(l \Delta t), \mathbf{w}_{x}(l \Delta t), \mathbf{w}_{y}(l \Delta t), \mathbf{s}(l \Delta t)\right] .
\end{aligned}
$$

Next $\mathbf{p}^{*}[(l+1) \Delta t], \mathbf{w}_{x}^{*}[(l+1) \Delta t]$, and $\mathbf{w}_{y}^{*}[(l+1) \Delta t]$ are used to estimate a value for the derivative functions $f_{p}, f_{x}$, and $f_{y}$ at the end of the time step, e.g.,

$$
\begin{aligned}
& f_{p}\left[i \Delta x, j \Delta y, \mathbf{p}[(l+1) \Delta t], \mathbf{w}_{x}[(l+1) \Delta t], \mathbf{w}_{y}[(l+1) \Delta t], \mathbf{s}[(l+1) \Delta t]\right] \simeq \\
& f_{p}\left[i \Delta x, j \Delta y, \mathbf{p}^{*}[(l+1) \Delta t], \mathbf{w}_{x}^{*}[(l+1) \Delta t], \mathbf{w}_{y}^{*}[(l+1) \Delta t], \mathbf{s}[(l+1) \Delta t]\right] .
\end{aligned}
$$

Then, in updating the fields, the derivatives at the beginning and end of the time step are averaged to approximate the derivative centered on the time step. Specifically the pressure field is advanced by the equation

$$
\begin{aligned}
& p[i \Delta x, j \Delta y,(l+1) \Delta t]=p[i \Delta x, j \Delta y, l \Delta t] \\
& +\frac{\Delta t}{2}\left\{f_{p}\left[i \Delta x, j \Delta y, \mathbf{p}(l \Delta t), \mathbf{w}_{x}(l \Delta t), \mathbf{w}_{y}(l \Delta t), \mathbf{s}(l \Delta t)\right]\right. \\
& \left.+f_{p}\left[i \Delta x, j \Delta y, \mathbf{p}^{*}[(l+1) \Delta t], \mathbf{w}_{x}^{*}[(l+1) \Delta t], \mathbf{w}_{y}^{*}[(l+1) \Delta t], \mathbf{s}[(l+1) \Delta t]\right]\right\} .
\end{aligned}
$$


The particle velocities are advanced in the same way.

The fourth-order Runge-Kutta method (Burden et al. 1981, Kreyszig 1988) is also widely used. We do not attempt a heuristic explanation of it here but rather just state the equations as they appear in the notation of this report:

$$
\begin{gathered}
p^{1}(i \Delta x, j \Delta y, l \Delta t)=\Delta t f_{p}\left[i \Delta x, j \Delta y, \mathbf{p}(l \Delta t), \mathbf{w}_{x}(l \Delta t), \mathbf{w}_{y}(l \Delta t), \mathbf{s}(l \Delta t)\right], \\
p^{2}(i \Delta x, j \Delta y, l \Delta t)=\Delta t f_{p}\left[i \Delta x, j \Delta y, \mathbf{p}(l \Delta t)+\mathbf{p}^{1}(l \Delta t) / 2,\right. \\
\left.\mathbf{w}_{x}(l \Delta t)+\mathbf{w}_{x}^{1}(l \Delta t) / 2, \mathbf{w}_{y}(l \Delta t)+\mathbf{w}_{y}^{1}(l \Delta t) / 2, \mathbf{s}[(l+1 / 2) \Delta t]\right] \\
p^{3}(i \Delta x, j \Delta y, l \Delta t)=\Delta t f_{p}\left[i \Delta x, j \Delta y, \mathbf{p}(l \Delta t)+\mathbf{p}^{2}(l \Delta t) / 2,\right. \\
\left.\mathbf{w}_{x}(l \Delta t)+\mathbf{w}_{x}^{2}(l \Delta t) / 2, \mathbf{w}_{y}(l \Delta t)+\mathbf{w}_{y}^{2}(l \Delta t) / 2, \mathbf{s}[(l+1 / 2) \Delta t]\right], \\
p^{4}(i \Delta x, j \Delta y, l \Delta t)=\Delta t f_{p}\left[i \Delta x, j \Delta y, \mathbf{p}(l \Delta t)+\mathbf{p}^{3}(l \Delta t),\right. \\
\left.\mathbf{w}_{x}(l \Delta t)+\mathbf{w}_{x}^{3}(l \Delta t), \mathbf{w}_{y}(l \Delta t)+\mathbf{w}_{y}^{3}(l \Delta t), \mathbf{s}[l \Delta t]\right]
\end{gathered}
$$

and

$$
\begin{aligned}
& p[i \Delta x, j \Delta y,(l+1) \Delta t]=p[i \Delta x, j \Delta y, l \Delta t] \\
& \quad+\frac{1}{6}\left\{\begin{array}{l}
p^{1}(i \Delta x, j \Delta y, l \Delta t)+2 p^{2}(i \Delta x, j \Delta y, l \Delta t) \\
+2 p^{3}(i \Delta x, j \Delta y, l \Delta t)+p^{4}(i \Delta x, j \Delta y, l \Delta t)
\end{array}\right\} .
\end{aligned}
$$

Equations for $w_{x}$ are the same except with $f_{x}$ replacing $f_{p}$ and $(i+1 / 2) \Delta x$ replacing $i \Delta x$; equations for $w_{y}$ are the same except with $f_{y}$ replacing $f_{p}$ and $(j+1 / 2) \Delta y$ replacing $j \Delta y$.

\section{Solution on a Grid Staggered in Time}

A common approach to FDTD solution for wave propagation in a nonmoving medium is to use a staggered, centered finite-differences grid in time. This approach is discussed, for example, by Yee (1966) and Botteldooren (1994). The main motivation for this approach is that it produces a solution with second-order accuracy with regard to the 
temporal grid resolution $\Delta t$, while requiring storage of the wavefield variables at only a single time step.

Consider Eq. 3-5, as specialized for a nonmoving medium:

$$
\begin{aligned}
& \frac{\partial p}{\partial t}=-\kappa\left(\frac{\partial w_{x}}{\partial x}+\frac{\partial w_{y}}{\partial y}\right)+\kappa Q, \\
& \frac{\partial w_{x}}{\partial t}=-b \frac{\partial p}{\partial x}+b F_{x}
\end{aligned}
$$

and

$$
\frac{\partial w_{y}}{\partial t}=-b \frac{\partial p}{\partial y}+b F_{y} .
$$

Suppose now that the pressure is stored on the whole time steps and the velocities at the half time steps. By forming centered FD approximations in time, we have, for the pressure

$$
\begin{aligned}
\frac{\partial p[i \Delta x}{\partial t \Delta y,(l+1 / 2) \Delta t]} & \\
\quad= & f_{p}\left[i \Delta x, j \Delta y, \mathbf{w}_{x}[(l+1 / 2) \Delta t], \mathbf{w}_{y}[(l+1 / 2) \Delta t], \mathbf{s}[(l+1 / 2) \Delta t]\right] \\
\simeq & \frac{p[i \Delta x, j \Delta y,(l+1) \Delta t]-p[i \Delta x, j \Delta y, l \Delta t]}{\Delta t}
\end{aligned}
$$

Solving for $p[i \Delta x, j \Delta y,(l+1) \Delta t]$, we have

$$
\begin{aligned}
& p[i \Delta x, j \Delta y,(l+1) \Delta t]=p[i \Delta x, j \Delta y, l \Delta t] \\
& \quad+\Delta t f_{p}\left[i \Delta x, j \Delta y, \mathbf{w}_{x}[(l+1 / 2) \Delta t], \mathbf{w}_{y}[(l+1 / 2) \Delta t], \mathbf{s}[(l+1 / 2) \Delta t]\right] .
\end{aligned}
$$

A similar process for the velocities yields

$$
\begin{aligned}
& w_{x}[(i+1 / 2) \Delta x, j \Delta y,(l+1 / 2) \Delta t]=w_{x}[(i+1 / 2) \Delta x, j \Delta y,(l-1 / 2) \Delta t] \\
& +\Delta t f_{x}[(i+1 / 2) \Delta x, j \Delta y, \mathbf{p}(l \Delta t), \mathbf{s}(l \Delta t)]
\end{aligned}
$$


and

$$
\begin{aligned}
& w_{y}[i \Delta x,(j+1 / 2) \Delta y,(l+1 / 2) \Delta t]=w_{y}[i \Delta x,(j+1 / 2) \Delta y,(l-1 / 2) \Delta t] \\
& +\Delta t f_{y}[i \Delta x,(j+1 / 2) \Delta y, \mathbf{p}(l \Delta t), \mathbf{s}(l \Delta t)] .
\end{aligned}
$$

Note, for this scheme, that $f_{p}$ is needed in Eq. 48 only on the half time steps, and that only the particle velocities (not the pressure) are needed for its calculation. Furthermore, in Eq. 49 and 50, $f_{x}$ and $f_{y}$ are needed only on the whole time steps and require only the pressure. Therefore, the solution can be advanced in time by solving for the pressure on a whole time step, solving for the particle velocities on the next half time step, solving for the pressure at the next whole time step, etc. The wavefield variables can be overwritten in place as they are updated. Because the centered differences are accurate to secondorder, this scheme provides good accuracy as well as efficiency in its use of computer memory. This staggered scheme is usually called the leap-frog method, which unfortunately is the same term used by Kasahara (1977) in connection with the unstaggered, centered, second-order scheme (Eq. 33).

Extending consideration now to the moving medium, we see in comparing Eq. 3 and 44 that pressure is no longer absent from $f_{p}$. Similarly $w_{x}$ and $w_{y}$ are required in the calculation of $f_{x}$ and $f_{y}$ :

$$
\begin{aligned}
& p[i \Delta x, j \Delta y,(l+1) \Delta t]=p[i \Delta x, j \Delta y, l \Delta t] \\
& +\Delta t f_{p}\left[\begin{array}{l}
i \Delta x, j \Delta y, \mathbf{p}[(l+1 / 2) \Delta t], \mathbf{w}_{x}[(l+1 / 2) \Delta t], \\
\mathbf{w}_{y}[(l+1 / 2) \Delta t], \mathbf{s}[(l+1 / 2) \Delta t]
\end{array}\right] .
\end{aligned}
$$

A similar process for the velocities yields

$$
\begin{gathered}
w_{x}[(i+1 / 2) \Delta x, j \Delta y,(l+1 / 2) \Delta t]=w_{x}[(i+1 / 2) \Delta x, j \Delta y,(l-1 / 2) \Delta t] \\
+\Delta t f_{x}\left[(i+1 / 2) \Delta x, j \Delta y, \mathbf{p}(l \Delta t), \mathbf{w}_{x}(l \Delta t), \mathbf{w}_{y}(l \Delta t), \mathbf{s}(l \Delta t)\right],
\end{gathered}
$$

and

$$
\begin{gathered}
w_{y}[i \Delta x,(j+1 / 2) \Delta y,(l+1 / 2) \Delta t]=w_{y}[i \Delta x,(j+1 / 2) \Delta y,(l-1 / 2) \Delta t] \\
+\Delta t f_{y}\left[i \Delta x,(j+1 / 2) \Delta y, \mathbf{p}(l \Delta t), \mathbf{w}_{x}(l \Delta t), \mathbf{w}_{y}(l \Delta t), \mathbf{s}(l \Delta t)\right] .
\end{gathered}
$$


As a result, it is no longer possible to implement the basic staggered-in-time scheme considered in the previous paragraph. One work-around that might be considered is to substitute $\{\mathbf{p}[(l+1) \Delta t]+\mathbf{p}[l \Delta t]\} / 2$ where $\mathbf{p}[(l+1 / 2) \Delta t]$ is needed in Eq. 48. However, one is then left with an equation for $p[i \Delta x, j \Delta y,(l+1) \Delta t]$ that cannot be solved without simultaneously knowing the pressure at all locations at $t=(l+1) \Delta t$. Therefore, this procedure does not provide an explicit method for updating the pressure field at each time step.

Another possibility would be to simply use the pressure field $\mathbf{p}(l \Delta t)$ in place of $\mathbf{p}[(l+$ $1 / 2) \Delta t$ when evaluating $f_{p}$, and $\mathbf{w}_{x}[(l-1 / 2) \Delta t]$ and $\mathbf{w}_{y}[(l-1 / 2) \Delta t]$ in place of $\mathbf{w}_{x}(l \Delta t)$ and $\mathbf{w}_{y}(l \Delta t)$ when evaluating $f_{x}$ and $f_{y}$. This nonrigorous procedure is somewhat similar to using the Euler (forward-difference) method from the previous section to evaluate the advective terms while maintaining the staggered-in-time grid approach for the terms related to the nonmoving medium. From a programming standpoint, one simply updates (overwrites) $\mathbf{p}$ based on calculation of $f_{p}$ from the currently stored $\mathbf{p}, \mathbf{w}_{x}$, and $\mathbf{w}_{y}$, and then updates $\mathbf{w}_{x}$ and $\mathbf{w}_{y}$ based on calculation of $f_{x}$ and $f_{y}$ from the currently stored values for $\mathbf{p}$, $\mathbf{w}_{x}$, and $\mathbf{w}_{y}$. This alternating process is repeated until the calculation reaches the desired end. The calculations in Salomons et al. (2002) and Blumrich and Heimann (2002) appear to be based on such a procedure. Salomons et al. mention (p. 486) that they first solve for the particle velocities and then use these updated velocities to calculate the pressure, cautioning that "Use of the non-updated velocities gives an unstable solution." Thus, the authors appear to be using a temporally staggered implementation, although it is not entirely clear from the equations presented in the text.

A rigorous scheme for performing centered, finite-difference calculations on a staggered-in-time grid has been proposed by D. Aldridge. ${ }^{*}$ This scheme involves storing the field variables back two steps in time, rather than just one, as has been considered thus far. Forming a centered finite difference across two time steps, one has, instead of Eq. 51,

$$
\begin{aligned}
& p[i \Delta x, j \Delta y,(l+1) \Delta t] \\
& \quad=p[i \Delta x, j \Delta y,(l-1) \Delta t] \\
& \quad+2 \Delta t f_{p}\left[i \Delta x, j \Delta y, \mathbf{p}(l \Delta t), \mathbf{w}_{x}(l \Delta t), \mathbf{w}_{y}(l \Delta t), \mathbf{s}(l \Delta t)\right] .
\end{aligned}
$$

The problem now is that the velocities are unknown on the time step $t=l \Delta t$. This can be overcome by averaging the velocities from the neighboring two time steps; that is,

\footnotetext{
*Personal communication, 2003.
} 


$$
\mathbf{w}_{x}(l \Delta t) \simeq \frac{\mathbf{w}_{x}[(l+1 / 2) \Delta t]+\mathbf{w}_{x}[(l-1 / 2) \Delta t]}{2}
$$

and

$$
\mathbf{w}_{y}(l \Delta t) \simeq \frac{\mathbf{w}_{y}[(l+1 / 2) \Delta t]+\mathbf{w}_{y}[(l-1 / 2) \Delta t]}{2}
$$

Similarly, the velocities can be updated with the equations

$$
\begin{gathered}
w_{x}[(i+1 / 2) \Delta x, j \Delta y,(l+1 / 2) \Delta t]=w_{x}[(i+1 / 2) \Delta x, j \Delta y,(l-3 / 2) \Delta t] \\
+2 \Delta t f_{x}\left[\begin{array}{l}
(i+1 / 2) \Delta x, j \Delta y, \mathbf{p}[(l-1 / 2) \Delta t], \mathbf{w}_{x}[(l-1 / 2) \Delta t], \\
\mathbf{w}_{y}[(l-1 / 2) \Delta t], \mathbf{s}[(l-1 / 2) \Delta t]
\end{array}\right], \\
w_{y}[i \Delta x,(j+1 / 2) \Delta y,(l+1 / 2) \Delta t]=w_{y}[i \Delta x,(j+1 / 2) \Delta y,(l-3 / 2) \Delta t] \\
+2 \Delta t f_{y}\left[\begin{array}{l}
i \Delta x,(j+1 / 2) \Delta y, \mathbf{p}[(l-1 / 2) \Delta t], \\
\mathbf{w}_{x}[(l-1 / 2) \Delta t], \mathbf{w}_{y}[(l-1 / 2) \Delta t], \mathbf{s}[(l-1 / 2) \Delta t]
\end{array}\right],
\end{gathered}
$$

and

$$
\mathbf{p}[(l-1 / 2) \Delta t] \simeq \frac{\mathbf{p}[l \Delta t]+\mathbf{p}[(l-1) \Delta t]}{2} .
$$

This scheme is similar to the unstaggered leap-frog method discussed in the previous section. The main differences are (1) the averaging of the field variables from the neighboring time steps, displaced $\pm 1 / 2$ time of the centered approximation, and (2) the alternating update of the pressure and particle velocities. 


\section{IMPLEMENTATION OF REFLECTIVE SURFACES}

In this section we consider boundary conditions for the finite-difference solution as well as the incorporation of a physically realistic ground layer.

\section{Rigid Surfaces}

Hard surfaces such as buildings, walls, and ice can often be modeled as acoustically rigid. By definition, the acoustic particle velocity vanishes in the direction normal to the rigid surface. A practical method for handling the rigid boundary is the method of images. In this method the actual medium below the rigid boundary is replaced by the mirror reflection of the medium above the boundary. An image source is placed at a distance below the boundary that is equal to the distance above the boundary for the actual source. Therefore, the solutions of the differential equations above and below the boundary are the same, except that they are reversed in the coordinate direction normal to the boundary. As a result, the acoustic particle velocities in the two solutions exactly cancel at the boundary. Because the solution above the boundary satisfies the original differential equation and the desired boundary condition, we are guaranteed that it is the correct, unique solution. (The solution below the boundary is incorrect, but that is not a concern.)

Let us consider the finite-difference equations $23-27$ in the presence of a rigid surface. ${ }^{*}$ The surface is assumed to be positioned at $y=(j-1 / 2) \Delta y$ in Figure 1. In the pressure derivative equation, Eq. 23, we of course would set $w_{y}[i \Delta x,(j-1 / 2) \Delta y, t]=0$. One also must have $v_{y}[i \Delta x,(j-1 / 2) \Delta y, t]=0$ at the rigid boundary. (Otherwise, the wind fields on the two sides of the boundary would be discontinuous.) The only other quantity in Eq. 23 that is directly affected by the boundary is $p[i \Delta x,(j-1) \Delta y, t]$. By the image method, the pressure field is reflected about the boundary, so that $p[i \Delta x,(j-1) \Delta y, t]=$ $p[i \Delta x, j \Delta y, t]$. Therefore, Eq. 23 becomes

\footnotetext{
"The term "surface" is used here, although, strictly speaking, the rigid condition in a 2-D code is implemented along a line.
} 


$$
\begin{aligned}
f_{p}\left[i \Delta x, j \Delta y, \mathbf{p}(t), \mathbf{w}_{x}(t), \mathbf{w}_{y}(t), \mathbf{s}(t)\right] \simeq \\
-\frac{1}{4 \Delta x}\left\{v_{x}[(i+1 / 2) \Delta x, j \Delta y, t]+v_{x}[(i-1 / 2) \Delta x, j \Delta y, t]\right\} \\
\quad \times\{p[(i+1) \Delta x, j \Delta y, t]-p[(i-1) \Delta x, j \Delta y, t]\} \\
-\frac{1}{4 \Delta y} v_{y}[\Delta x,(j+1 / 2) \Delta y, t] \\
\quad \times\{p[i \Delta x,(j+1) \Delta y, t]-p[i \Delta x, j \Delta y, t]\} \\
-\kappa(i \Delta x, j \Delta y, t)\left\{\frac{w_{x}[(i+1 / 2) \Delta x, j \Delta y, t]-w_{x}[(i-1 / 2) \Delta x, j \Delta y, t]}{\Delta x}\right. \\
\left.+\frac{w_{y}[i \Delta x,(j+1 / 2) \Delta y, t]}{\Delta y}\right\} \\
+\kappa(i \Delta x, j \Delta y, t) Q(i \Delta x, j \Delta y, t) .
\end{aligned}
$$

Similarly Eq. 27 has four terms involving $y$ components of the acoustic particle velocity and wind at $y=(j-1 / 2) \Delta y$, all of which are zero. Also needed are the $x$ components of the acoustic particle velocity and wind at $y=(j-1) \Delta y$. But because the $x$ coordinate runs parallel to the boundary, the fields for these quantities are also reflected, so $w_{x}[(i+1 / 2) \Delta x,(j-1) \Delta y, t]=w_{x}[(i+1 / 2) \Delta x, j \Delta y, t]$ and $v_{x}[(i+1 / 2) \Delta x,(j-1) \Delta y, t]=v_{x}[(i$ $+1 / 2) \Delta x, j \Delta y, t]$. Finally, in Eq. 27 there are two terms involving $y$ components of the velocities at $y=(j-1 / 2) \Delta y$ that must be set to zero.

\section{Sound Propagation in a Porous Medium and Implementation}

Most common outdoor ground surfaces cannot be modeled satisfactorily as ideal, rigid surfaces. This is because sound energy propagates into the pores of the ground, where it is dissipated by viscosity and thermal conduction. Ground surfaces with relatively large, open pores, such as snow, absorb much of the sound energy incident upon them. Surfaces with small pores, such as cement and asphalt, reflect most of the energy. The acoustical behavior of soils is intermediate between these extremes. For completeness, we therefore consider in this report FDTD implementation of a porous ground layer. We show that the ground can be modeled with equations very similar to those of the air.

Morse and Ingard (1968, Eq. 6.2.22 and 6.2.23) propose the following phenomenological equations for sound propagation in a porous medium: 


$$
\begin{gathered}
\frac{\partial p}{\partial t}=-\left(\kappa_{p} / \Omega\right) \nabla \cdot \mathbf{w}, \\
\rho_{p} \frac{\partial \mathbf{w}}{\partial t}+\sigma \mathbf{w}=-\nabla p,
\end{gathered}
$$

where $\sigma$ is the static flow resistivity, $\kappa_{p}$ is the bulk modulus of the air in the pores, $\rho_{p}$ is the density of air in the pores, and $\Omega$ is the porosity (void fraction) of the medium. The bulk modulus is bounded by its isothermal value $\rho c^{2} / \gamma$ and its adiabatic value $\rho c^{2}$ (where $c$ indicates the adiabatic sound speed), with isothermal conditions prevailing for small pores and/or low frequencies. Subsequent authors, such as Attenborough (1983), have shown that when the properties of the material are considered in bulk, $\rho_{p}$ has a complicated frequency dependence and in fact becomes a complex quantity in the frequency domain. At low frequencies, $\rho_{p}$ may be replaced by $s_{c} / \Omega$, where $s_{c}$ is called the structure constant or effective density factor. Furthermore, $s_{c}$ can be related to the tortuosity factor $q$, which describes the slanting of the pores. (For pores at a fixed slant angle $\theta, q$ is simply $1 / \cos \theta$.) The relationship between $s_{c}$ and $q$ can be determined for some idealized cases, such as circular cylindrical pores, for which $s_{c}=(4 / 3) q^{2} / \Omega$ (Attenborough 1983). However, the relationship between $s_{c}$ and $q$ depends, in general, on the geometry of the pores.

Restricting our attention to frequencies low enough that $\rho_{p}$ can be considered a real constant and the bulk modulus is isothermal, we may rewrite Eq. 61 and 62 slightly to produce the following model equations for the porous medium:

$$
\begin{aligned}
& \frac{\partial p}{\partial t}=-\kappa_{e} \nabla \cdot \mathbf{w}, \\
& \rho_{e} \frac{\partial \mathbf{w}}{\partial t}+\sigma \mathbf{w}=-\nabla p,
\end{aligned}
$$

where

$$
\kappa_{e}=\rho c^{2} / \gamma
$$

and

$$
\rho_{e}=s_{c} \rho / \Omega
$$


are the effective bulk modulus and density of the fluid saturating the porous material, respectively. This equation set is nearly the same as the one used by Salomons et al. (2002), except that they use $\kappa_{e}=\rho c^{2} / \Omega$, which would apply to adiabatic, as opposed to isothermal, conduction in the pores.

The main distinction between Eq. 63 and 64 and those for the nonmoving fluid is the presence of the term involving the static flow resistivity, $\sigma$. Physically this term implies that a static pressure gradient will not accelerate the fluid to an infinite speed; rather, the fluid velocity approaches an equilibrium that is determined by the viscous drag of the porous frame. Since $\Omega$ is always less than one and $s_{c}$ is always greater than 1 , the effective density $\rho_{e}$ is always greater than its value in the fluid. The phase speed, $\sqrt{\kappa_{e} / \rho_{e}}=c / \sqrt{\gamma s_{c}}$, is always less than $c$, implying that the wavelength is shorter in the porous ground medium than in the air. Table 1 shows some typical parameter values for porous media. Table 1. Typical values of the static flow resistivity,
porosity, and tortuosity for common porous
ground surfaces.

\begin{tabular}{|lccc|}
\hline Material & $\begin{array}{c}\text { Flow resistivity, } \sigma \\
\left(\mathbf{P a - s} \mathbf{~ m}^{-2}\right)\end{array}$ & Porosity, $\Omega$ & Tortuosity, $q$ \\
\hline Asphalt & $3 \times 10^{7}$ & 0.1 & 3.2 \\
Grass & $2 \times 10^{5}$ & 0.5 & 1.4 \\
Forest & $1 \times 10^{5}$ & 0.6 & 1.3 \\
Sand & $5 \times 10^{4}$ & 0.35 & 1.6 \\
Snow & $1 \times 10^{3}$ & 0.6 & 1.7 \\
\hline
\end{tabular}

Finite-difference implementation of Eq. 63 is the same as an implementation for a nonmoving medium. Implementation of Eq. 64 is slightly different. Let us begin by rewriting it in component form as

$$
\begin{aligned}
& \frac{\partial w_{x}}{\partial t}=-s_{e} w_{x}-b_{e} \frac{\partial p}{\partial x}, \\
& \frac{\partial w_{y}}{\partial t}=-s_{e} w_{y}-b_{e} \frac{\partial p}{\partial y},
\end{aligned}
$$

where $s_{e}=\sigma / \rho_{e}$ and $b_{e}=1 / \rho_{e}$. On the spatially staggered grid, we would approximate Eq. 67 with a finite difference centered at $x=(i+1 / 2) \Delta x$ and $y=j \Delta y$, as discussed in Section 3. Similarly the approximation for Eq. 68 would be centered at $x=i \Delta x$ and $y=(j+1 / 2) \Delta y$. 
Assuming $b_{e}$ and $s_{e}$ are defined at the pressure grid nodes, we can determine these quantities at the nodes where they are needed using Eq. 6 and 7. The pressure gradients follow from Eq. 10 and 11 as before. The velocities in the terms involving $s_{e}$ are known directly at the approximation centers. We see that FDTD implementation of the porous layer is trivial once the basic procedure for the moving medium has been worked out.

\section{Absorbing Boundary Condition}

Most FDTD codes use artificial absorbing layers (called absorbing boundary conditions, or ABCs) around the edge of the domain to avoid spurious numerical reflections at the edges. Popular methods include the Cerjan attenuating layer (Cerjan et al. 1985) and, more recently, the perfectly matched layer (Berenger 1994, Yuan et al. 1997). An interesting question is whether a porous layer of the type discussed in the previous section can be used as an $\mathrm{ABC}$. As a starting point, consider the reflection coefficient for waves in a fluid incident upon a solid material (e.g., Kinsler et al. 1982):

$$
R=\frac{Z_{c} \cos \theta_{i}-\rho c \cos \theta_{t}}{Z_{c} \cos \theta_{i}+\rho c \cos \theta_{t}}
$$

where $Z_{c}$ is the characteristic impedance for waves propagating in the solid, $\theta_{i}$ is the angle of incidence, and $\theta_{t}$ is the angle of transmission. The angles $\theta_{i}$ and $\theta_{t}$ are measured relative to the surface normal. Note that $\theta_{t}$ is, in general, complex, with the imaginary part being associated with evanescent interface waves. If the reflection coefficient is to be zero, we must therefore have

$$
\frac{Z_{c}}{\rho c}=\frac{\cos \theta_{t}}{\cos \theta_{i}}
$$

In addition, Snell's law implies that

$$
k \sin \theta_{i}=k_{c} \sin \theta_{t}
$$

where $k=\omega / c$ is the wavenumber in the fluid and $k_{c}$ is the complex wavenumber in the solid. Using Snell's law to eliminate $\cos \theta_{t}$ from Eq. 70 results in

$$
\left(\frac{Z_{c}}{\rho c}\right)^{2} \cos ^{2} \theta_{i}+\left(\frac{k}{k_{c}}\right)^{2} \sin ^{2} \theta_{i}=1
$$


Expressions for the characteristic impedance and complex wavenumber can be derived directly from Eq. 63 and 64. We look for plane-wave solutions to these equations and therefore set

$$
p=A e^{i\left(k_{c} x-\omega t\right)}, w_{x}=B e^{i\left(k_{c} x-\omega t\right)}, w_{y}=w_{z}=0
$$

Such a solution represents waves traveling in the $+x$ direction. By substitution into Eq. 63 and 64, we find

$$
\frac{A}{B}=\frac{k_{c} \kappa_{e}}{\omega}=\frac{\omega \rho_{c}}{k_{c}}
$$

where $\rho_{c}=\rho_{e}+i \sigma / \omega$ is called the complex density. Solving the preceding equations for the complex wavenumber, we have

$$
k_{c}=\omega \sqrt{\frac{\rho_{c}}{\kappa_{e}}} .
$$

By definition, $Z_{c}=A / B$, which is now determined as

$$
Z_{c}=\sqrt{\kappa_{e} \rho_{c}}
$$

Substitution into Eq. 72 now yields

$$
\frac{\rho_{c} \kappa_{e}}{\rho^{2} c^{2}} \cos ^{2} \theta_{i}+\frac{\kappa_{e}}{\rho_{c} c^{2}} \sin ^{2} \theta_{i}=1
$$

If we were to set $\kappa_{e}=\rho c^{2}$ and $\rho_{c}=\rho$ in the porous layer, then Eq. 77 would be an identity for all angles of incidence. This outcome is to be expected, since, if the air layer and porous medium have identical properties, there is in effect no interface. More interesting is what happens when there is attenuation in the porous medium and $\rho_{c}$ is complex. Writing out the real and imaginary components of Eq. 77 explicitly, we have

$$
\frac{\left(\rho_{e}+i \sigma / \omega\right) \kappa_{e}}{\rho^{2} c^{2}} \cos ^{2} \theta_{i}+\frac{\kappa_{e}\left(\rho_{e}-i \sigma / \omega\right)}{\left(\rho_{e}^{2}+\sigma^{2} / \omega^{2}\right) c^{2}} \sin ^{2} \theta_{i}=1 .
$$


If $\sigma / \omega$ is very small in comparison to $\rho_{e}$, this relationship can be satisfied approximately for all $\theta_{i}$ by setting $\kappa_{e}=\rho c^{2}$ and $\rho_{e}=\rho$, or equivalently $s_{c}=\Omega=\gamma=1$. Thus, we may use a porous layer as an $\mathrm{ABC}$ so long as the artificial static flow resistivity is small enough to satisfy the relationship $\sigma / \omega \rho<<1$ throughout the frequency range of interest. 


\section{IMPLEMENTATION AND EXAMPLE CALCULATIONS}

\section{Numerical Issues}

Dependence of time step on Mach number

For numerical stability the time step must satisfy

$$
\Delta t<\frac{\Delta r}{u}
$$

where $u$ is the speed at which the energy propagates and $\Delta r$ the grid spacing. On a twodimensional grid, $\Delta r=1 / \sqrt{(\Delta x)^{-2}+(\Delta y)^{-2}}$. In the FDTD literature, it is common to define the Courant number as

$$
C=\frac{u \Delta t}{\Delta r}
$$

Therefore, to satisfy Eq. 79, we must satisfy the so-called Courant condition, $C<1$. The time step and grid spacing must be chosen with this constraint in mind. Since the grid spacing should generally be a small fraction of a wavelength for good numerical accuracy, the Courant condition in practice imposes a limitation on the maximum time step possible for stable calculations. It should also be kept in mind that satisfaction of the Courant condition does not ensure accurate calculations.

Let us consider the implications of the Courant condition for propagation in a uniform flow. In this case, $u$ is determined by a combination of the sound speed and wind velocity. In the downwind direction, we have $u=u_{+}=c+v$. In the upwind direction, $u=$ $u_{-}=c-v$. The wavelengths in these two directions are $\lambda_{+}=(c+v) / f$ and $\lambda_{-}=(c-v) / f$, respectively, where $f$ is the frequency. Since the wavelength is shortest in the upwind

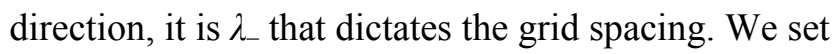

$$
\Delta r=\frac{\lambda}{N}=\frac{\lambda}{N}(1-M)
$$

where $N$ is the number of grid points per wavelength, $M=v / c$ is the Mach number, and $\lambda$ $=c / f$ is the wavelength for the medium at rest. We see that a finer grid is required as $M$ increases. Regarding the time step, the Courant condition implies 
$\Delta t<\frac{\lambda}{N u}$

This condition is most difficult to meet when $u$ is largest, which is the case in the downwind direction. Therefore, we must use $u_{+}$in the preceding inequality if we are to have accurate results throughout the domain; specifically, we must set

$$
\Delta t<\frac{\lambda-}{N u_{+}}=\frac{1}{N f} \frac{1-M}{1+M}
$$

From this equation it follows that the time step at $M=1 / 3$ must be $1 / 2$ the value necessary at $M=0$. At $M=2 / 3$, the time step must be $1 / 5$ the value at $M=0$. And at $M=1$, a vanishing time step is required. The shortening of the required time step and grid spacing combine to make calculations at large Mach numbers more computationally intensive.

\section{Algorithm discussion}

The various algorithms in Section 4 differ in their memory requirements and number of calculations required per time step. In the following we consider the computational aspects of each algorithm individually.

Euler method, Eq. 29-31. This method is first order in $\Delta t$ and involves one calculation of the time-derivative functions $f_{p}, f_{x}$, and $f_{y}$ per time step. Regarding memory usage, the fields themselves must be stored, and also the calculations of the time-derivative functions at each grid point. Although one might initially think that the fields can be updated in situ while calculating the time-derivative functions on a grid-point-by-gridpoint basis (thereby not requiring storage of at every grid point), it must be remembered that the derivatives depend on neighboring field points as well as the FD center of the approximation. Therefore, if the fields at nearby grid points have been overwritten, the calculations of $f_{p}, f_{x}$, and $f_{y}$ will be incorrect. We characterize the calculation burden of the Euler method as one "unit" per time step, where one calculation unit is defined as the time necessary for a single evaluation of the time-derivative functions. The memory usage is two "units" per time step, where a single memory unit is defined as the memory required to store all of the fields at each grid point.

Unstaggered leap-frog scheme, Eq. 33. Since it is based on centered approximations, this method is second order in $\Delta t$. It involves one calculation of the time-derivative functions $f_{p}, f_{x}$, and $f_{y}$ per time step. This method is readily implemented by storing the fields over two time steps $(j$ and $j-1)$ and the time derivative functions at one time step $(j)$. Actually, a clever algorithm could avoid the need for calculating and storing $f_{p}, f_{x}$, and $f_{y}$ at each grid point, since the fields at time step $j-1$ can be overwritten without affecting the calculation of $f_{p}, f_{x}$, and $f_{y}$ at time step $j$. But such a procedure would introduce, in 
practice, more complicated memory operations. So we characterize the unstaggered leapfrog scheme as having a calculation burden of one unit per time step and memory usage of two to three units per time step, depending on the implementation.

Runge-Kutta 2nd order, Eq. 34-38. As indicated by the name, this method is second order in $\Delta t$. Two calculations of $f_{p}, f_{x}$, and $f_{y}$ per time step are needed per time step. In addition to storing the fields (which can be updated in situ) and time-derivative functions at each time step, one must store the time-derivative functions estimated for the end of the time step. Therefore, this method has a computational burden of two units and memory usage of three units per time step.

Runge-Kutta 4th order, Eq. 39-43. This method is fourth order in $\Delta t$. Four calculations of $f_{p}, f_{x}$, and $f_{y}$ are needed per time step. In addition to storing the fields (which can be updated in situ), four sets of time-derivative functions are needed per time step.

Actually, if one calculates the four time-derivative functions sequentially and updates the fields after each update, only three sets of arrays encompassing the computational grid need to be stored. Therefore, this method has a computational burden of four units and memory usage of three to five units per time step, depending on the implementation.

Staggered leap-frog scheme, Eq. 51-53 (and discussion in the paragraph after these equations). This method is second order in $\Delta t$ for the terms not involving motion in the propagation medium and first order in $\Delta t$ for the terms involving motion. It is the same as the Euler scheme in its computational and memory requirements.

Aldridge staggered scheme, Eq. 54-59. This method uses centered differences for all terms to achieve second-order accuracy in $\Delta t$. One evaluation of the time-derivative functions is required for each whole time step. The method is most readily implemented by storing the complete fields over two time steps, with additional memory needed to store the complete time-derivative functions at one time step. A reduction in memory usage is possible by observing that when the velocities are updated, the velocities are only needed at one previous time step; similarly, when the pressure is updated, the pressure is needed only at one previous time step. Therefore, some overwriting is possible. This method has a calculation burden of one unit per time step and memory usage of two to three units per time step, depending on the implementation.

The results of this discussion are summarized in Table 2. In comparing the methods, it should be kept in mind that the Euler method is in actuality unstable and therefore cannot really be considered. The staggered leap-frog scheme is also of questionable validity. One might be tempted to rule out the Runge-Kutta second-order method, since it requires twice as many calculations per time step as the unstaggered leap-frog and the Aldridge staggered methods. However, methods of the same temporal order do not necessarily possess the same accuracy. If one can take time steps with the second-order RungeKutta that are twice as long while maintaining the same accuracy as the unstaggered leapfrog and the Aldridge staggered methods, then the additional computational burden per 
time step would be worthwhile. Therefore, a more careful study of the properties of the methods is required. We will consider some empirical comparisons between the various methods later in this section. A more rigorous theoretical analysis is not considered in this report, however.

Table 2. Comparison of some methods for advancing in time the equations of sound propagation in a moving medium. One unit of memory usage is defined as the storage required for arrays of all the field components (acoustic pressure and particle velocities) at all grid nodes. One unit of computational burden is defined as the number of operations required to evaluate the time-derivative functions once for all of the fields.

\begin{tabular}{|lccc|}
\hline \multicolumn{1}{|c}{ Method } & Order in $\Delta t$ & Memory usage & Computational burden \\
\hline Euler & 1 & 2 & 1 \\
Unstaggered leap-frog & 2 & $2-3$ & 1 \\
Runge-Kutta, second-order & 2 & 3 & 2 \\
Runge-Kutta, fourth-order & 4 & $3-5$ & 4 \\
Staggered leap-frog & $1-2$ & 2 & 1 \\
Aldridge staggered & 2 & $2-3$ & 1 \\
\hline
\end{tabular}

\section{Implementation as a Matlab code}

We have implemented the two-dimensional propagation equations and numerical methods described in Sections 3-5 in a serial-processing Matlab code. The code provides a convenient environment for testing numerical methods for FDTD calculations in a moving medium. ${ }^{*}$ The time derivatives of the fields (as given by Eq. 23, 26, and 27) are implemented explicitly as separate functions, allowing all of the temporal finitedifference schemes discussed in Section 4 to be implemented with ease.

Spatial finite differencing is accomplished in the code using the Matlab circshift function, which circularly shifts numerical arrays upward, downward, rightward, or leftward. For example, a circular shift downward would move all rows down one position in the array, except for the last row, which is moved to the top of the array. In the finite difference code, this means that a wave propagating downward through the lower domain boundary reappears at the upper boundary. This non-physical behavior must be kept in mind when determining the total time of a simulation, so that spurious "wrap-around"

\footnotetext{
*A full 3-D, Fortran code that implements all of the important features in the 2-D Matlab code is now under development. The 3-D code performs calculations in parallel by segmenting the domain into rectangular-shaped boxes and using MPI (Message Passing Interface) instructions to exchange fields at common grid boundaries.
} 
solutions are not present. Alternatively an absorbing porous layer may be inserted in the vicinity of a boundary to prevent the wrap-around.

The 2-D Matlab code has also been written to accept user-specified perfectly rigid and acoustically porous objects in arbitrary number and positions. Technically the model for rigid surfaces in Section 5 assumed a surface of infinite extent. However, the code will allow users to specify finite-sized rigid objects. Corners are handled by applying the infinite-surface model right up to the corner.

\section{No Flow with Rigid Boundary}

To test the FDTD code and rigid-boundary implementation, we consider in this section a set of calculations for a harmonic source above a rigid boundary in a stationary medium. Analytical solutions to this problem are known. Specifically, for a point source radiating into an infinite 2-D medium (or equivalently a cylinder radiating into a 3-D medium), Morse and Ingard (1968, Sec. 7.3) show

$$
\mathbf{p}(x, y)=\frac{i}{4} H_{0}^{(1)}\left(k \sqrt{\left(x-x_{s}\right)^{2}+\left(y-y_{s}\right)^{2}}\right),
$$

where $i=\sqrt{-1},\left(x_{s}, y_{s}\right)$ is the source position, $k=2 \pi f / c$ is the wavenumber, $f$ is the source frequency, and $H_{0}^{(1)}$ is the zeroth-order Hankel function. The bolding of $\mathbf{p}$ indicates the complex phasor of the pressure field, as opposed to its real value. The rigid surface can be handled by the method of images (Morse and Ingard 1968, Sec. 7.4), yielding

$$
\begin{gathered}
\mathbf{p}(x, y)=\frac{i}{4} H_{0}^{(1)}\left(k \sqrt{\left(x-x_{s}\right)^{2}+\left(y-y_{s}\right)^{2}}\right) \\
+\frac{i}{4} H_{0}^{(1)}\left(k \sqrt{\left(x-x_{s}\right)^{2}+\left(y+y_{s}\right)^{2}}\right) .
\end{gathered}
$$

In the simulations, we implement the harmonic source with a finite duration signal that is tapered at the initiation and termination. The tapering alleviates numerical inaccuracies that are present when there is an abrupt change in the source emission. A cosine window taper is used here, as suggested to the authors by N. Symons. The tapered source equation is

$$
Q(t)=\left\{\begin{array}{cc}
(A / 2)\left[1-\cos \left(\pi t / T_{1}\right)\right] \cos (2 \pi f+\phi), & 0 \leq t<T_{1}, \\
A \cos (2 \pi f+\phi), & T_{1} \leq t \leq T-T_{2}, \\
(A / 2)\left[1+\cos \left(\pi(t-T) / T_{2}\right)\right] \cos (2 \pi f+\phi), & T-T_{2}<t \leq T, \\
0, & \text { otherwise. }
\end{array}\right.
$$


In the preceding equation, $A$ is the source amplitude, $\phi$ is the source phase, $T_{1}$ is the duration of the initiation taper, and $T_{2}$ is the duration of the termination taper. All calculations in this report use tapering over an interval of three periods in the harmonic wave $\left(T_{1}=T_{2}=3 / f\right)$.

The domain configuration for the no-flow calculations is shown in Fig. 2. The overall dimensions of the domain are $200 \mathrm{~m}$ in the horizontal and $100 \mathrm{~m}$ in the vertical. The origin is taken to be the middle of this domain. A $100-\mathrm{Hz}$ source is placed at $(-60 \mathrm{~m},-30$ $\mathrm{m}$ ). A rigid surface is present along the entire lower grid boundary. Absorbing layers, 20 $\mathrm{m}$ thick, are present at the top and left sides of the computational grid. These absorbing layers have $s_{c}=\Omega=\gamma=1$ as suggested earlier. The static flow resistivity is tapered from $100 \mathrm{~Pa}-\mathrm{s} \mathrm{m}^{-2}$ at the side of the layer closest to the source to $1000 \mathrm{~Pa}-\mathrm{s} \mathrm{m}^{-2}$ at the other side.

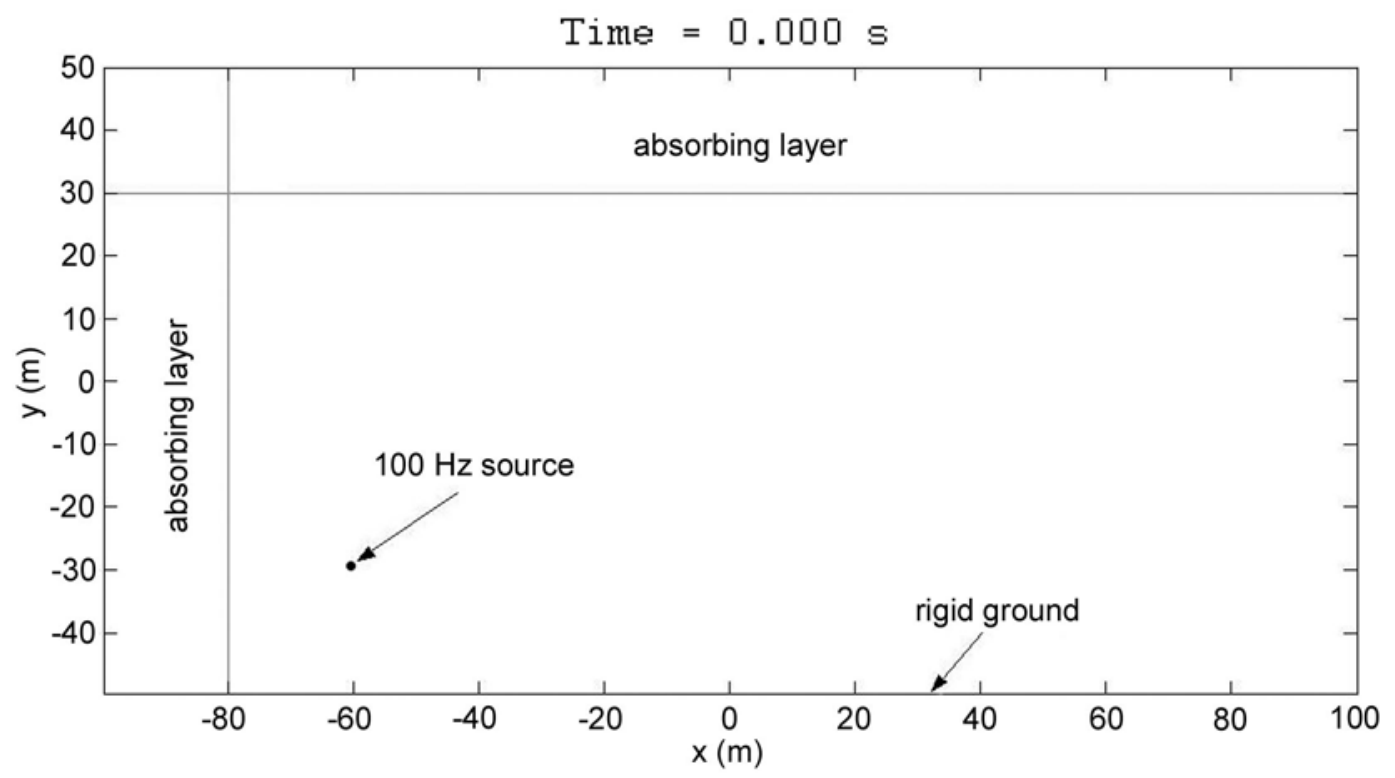

Figure 2. Configuration of the computational domain for calculations of the field generated by a harmonic source above a rigid boundary.

The calculations in this section use the second-order spatial differencing scheme discussed earlier in this report, together with a fourth-order Runge-Kutta scheme for marching the solution forward in time. Two numerical grid resolutions are considered: a low-resolution grid with $600 \times 300$ points and a high-resolution grid with $1200 \times 600$ points. Since the wavelength of sound at $100 \mathrm{~Hz}$ is $3.4 \mathrm{~m}$ in air, about 10 grid points per wavelength have been used in the low-resolution grid and 20 grid points per wavelength in the high-resolution grid. The Courant number (Eq. 80) was set to 0.8, leading to a time step of $0.58 \mathrm{~ms}$ for the low-resolution grid and $0.29 \mathrm{~ms}$ for the high-resolution grid. 
Figure 3 shows the pressure field $0.450 \mathrm{~s}$ after initiation of the $100-\mathrm{Hz}$ source. At this time, the waves have propagated approximately $150 \mathrm{~m}$ from the source in the $+x$ direction. Rapid attenuation of the sound energy is seen in the other directions, where absorbing layers are present. A series of spoke-like pressure minima radiating outward from the source are clearly evident. These are caused by destructive interference between the direct waves and the waves reflected from the rigid lower boundary.

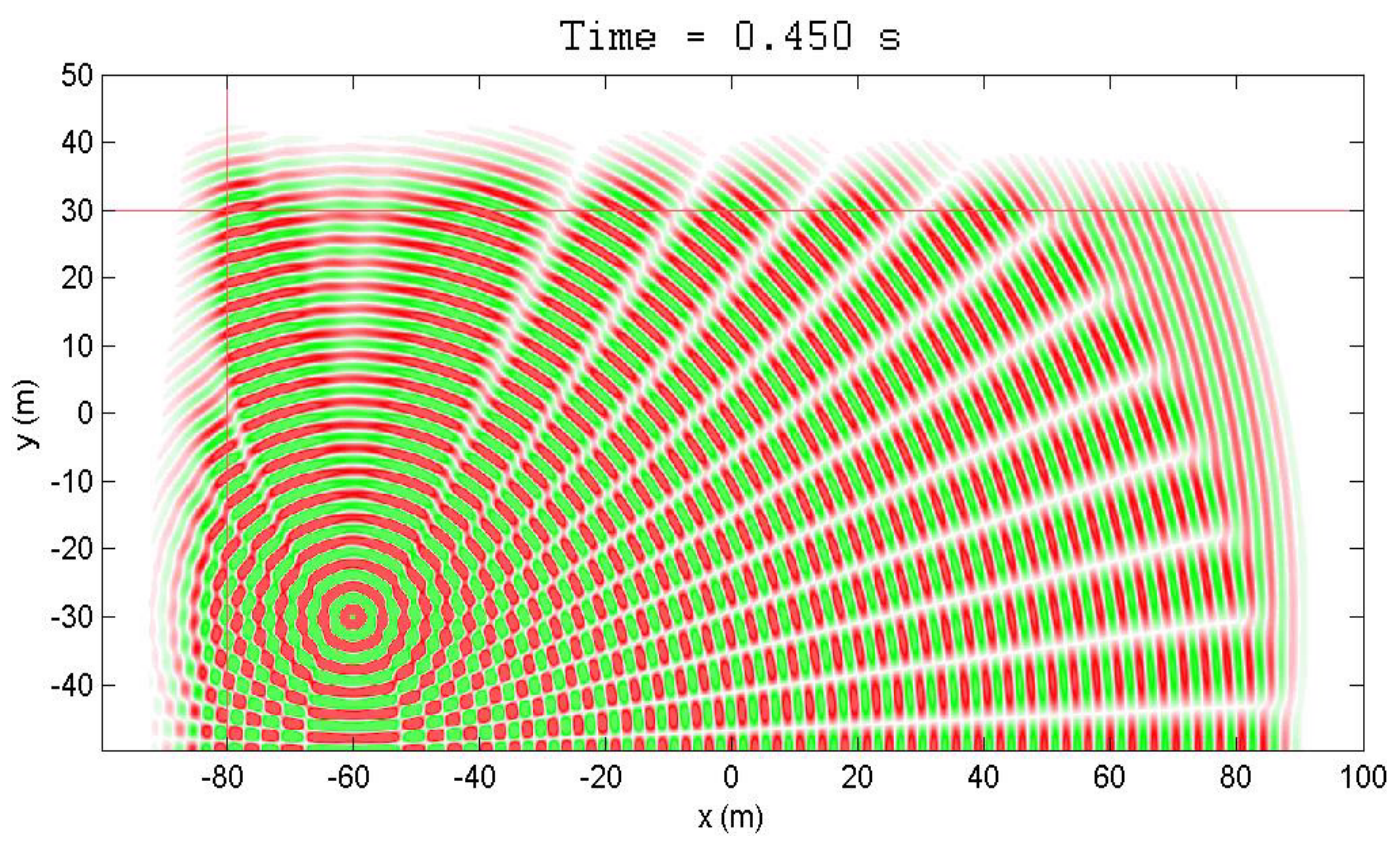

Figure 3. Sound pressure field $0.450 \mathrm{~s}$ after initiation of the $100-\mathrm{Hz}$ sound source.

Comparisons of calculated sound transmission loss (TL) ${ }^{*}$ to the theoretical result, Eq. 85, are presented in Fig. 4 and 5. These figures show results for the low- and highresolution grids, respectively. The receiver in this case is at the same height as the source, and the TL is plotted for locations increasing in horizontal distance from the source. The "dips" in the TL result from the interference minima mentioned in the preceding paragraph. The sound-pressure amplitude needed for the TL calculations was determined by locating the maximum absolute value of the pressure within the final 30 time steps (one to two wave periods) of the received signal. Very close agreement is obtained between the theory and the high-resolution FDTD calculations. The interference minima in the low-resolution calculations are shifted to noticeably smaller ranges than predicted by the theory.

"Sound transmission loss is the ratio of the pressure amplitude recorded at the receiver to the hypothetical value that would be observed in free space at a distance of $1 \mathrm{~m}$ from the source. The TL is expressed in decibels by taking the base-ten logarithm of the ratio and multiplying by 20. 


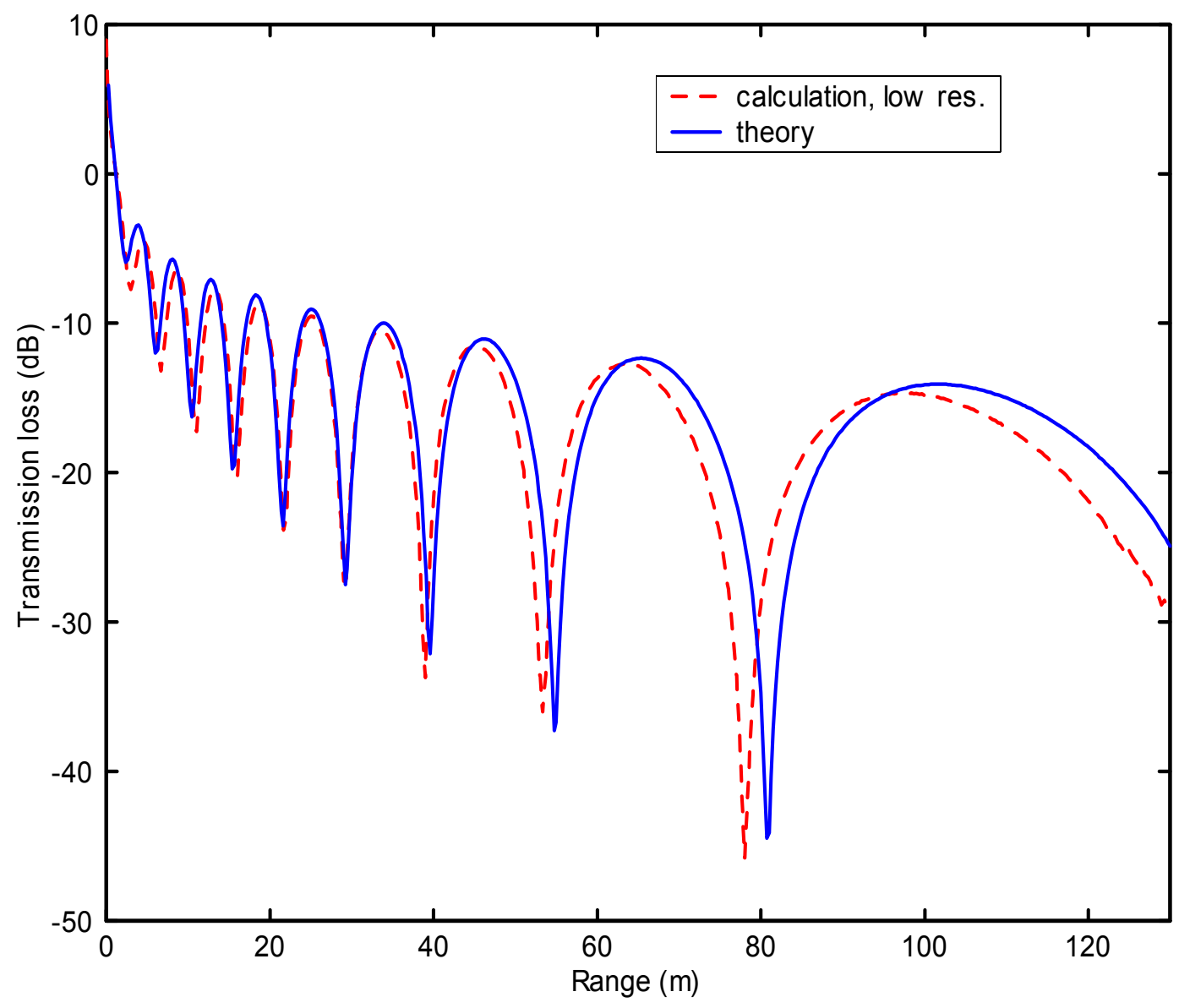

Figure 4. Comparison of the numerically calculated sound transmission loss with the theoretical result for the low-resolution numerical grid. 


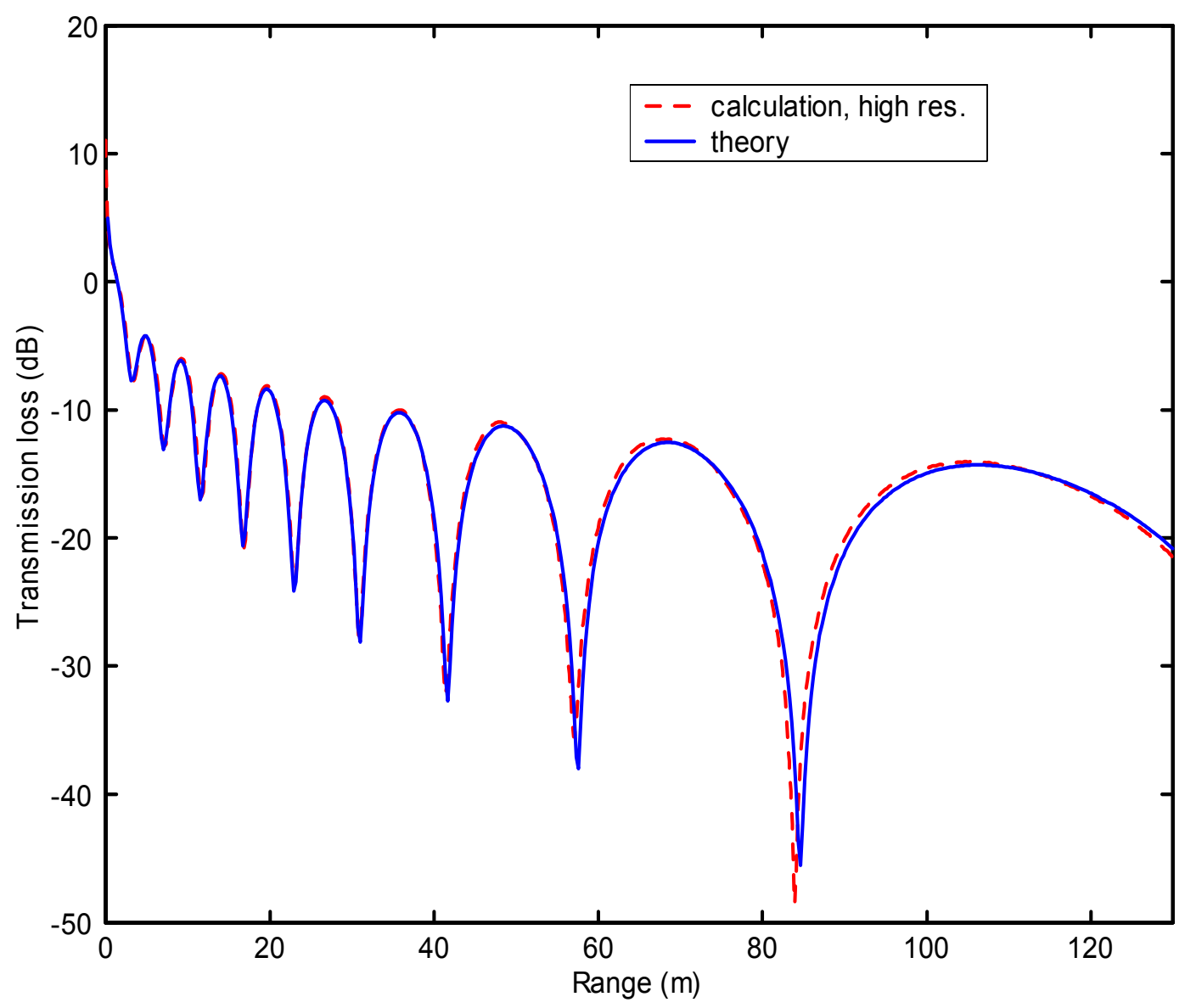

Figure 5. Comparison of the numerically calculated sound transmission loss with the theoretical result for the high-resolution numerical grid.

\section{Propagation over Porous Ground}

We next consider FDTD calculations with a porous ground model given by Eq. 63 and 64. The domain configuration (Fig. 6) is similar to the one used for the no-flow calculations (Fig. 2), except that the source has been moved to the center of the domain, a rigid barrier has been placed $20 \mathrm{~m}$ to the right of the source, and a 20 -m-thick ground layer is now present at the bottom of the domain. The absorbing layer to the left of the source has been removed. The source in the simulation emits 10 cycles of a $100-\mathrm{Hz}$ sine wave, with tapering applied during the three cycles after initiation and preceding termination. The rigid barrier is implemented using the surface boundary condition described for rigid surfaces in Section 5. 


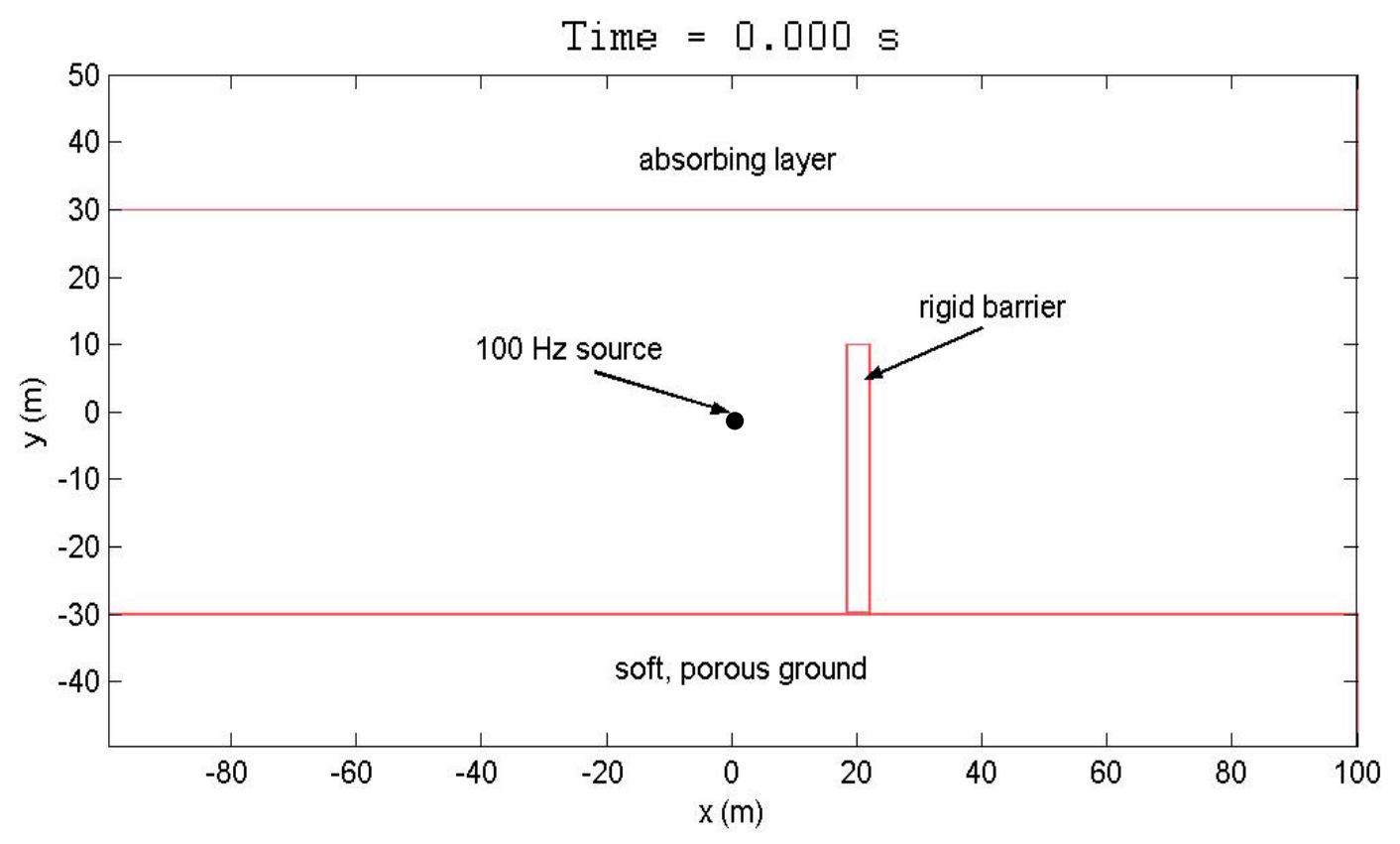

Figure 6. Configuration of the computational domain for calculations involving porous ground and a rigid barrier.

Figures 7-9 show calculations for an acoustically very soft surface. The porous material parameters are $\sigma=100 \mathrm{~Pa}-\mathrm{s} \mathrm{m}^{-2}, q=1.8$, and $\Omega=0.5$. In the first figure of the sequence, taken $0.057 \mathrm{~s}$ after source initiation, the sound waves are just beginning to impinge on the barrier. By the second figure (Fig. 8), taken at $0.154 \mathrm{~s}$, a wavetrain is seen reflecting strongly off the barrier while a second, weaker wavetrain reflects from the ground. A diffracted wavetrain curls around the barrier. The shorter wavelength of the sound and strong attenuation within the ground are readily evident. In Figure 9, taken at $0.250 \mathrm{~s}$, the initial wave and barrier reflection are clearly seen propagating leftward. The wavetrain that propagated over the top of the barrier (including the diffracted wave) propagates to the right. Weak ground reflections associated with each of these three wavetrains are also visible. Waves in the ground refract strongly toward the surface normal, as is expected from Snell's law. 


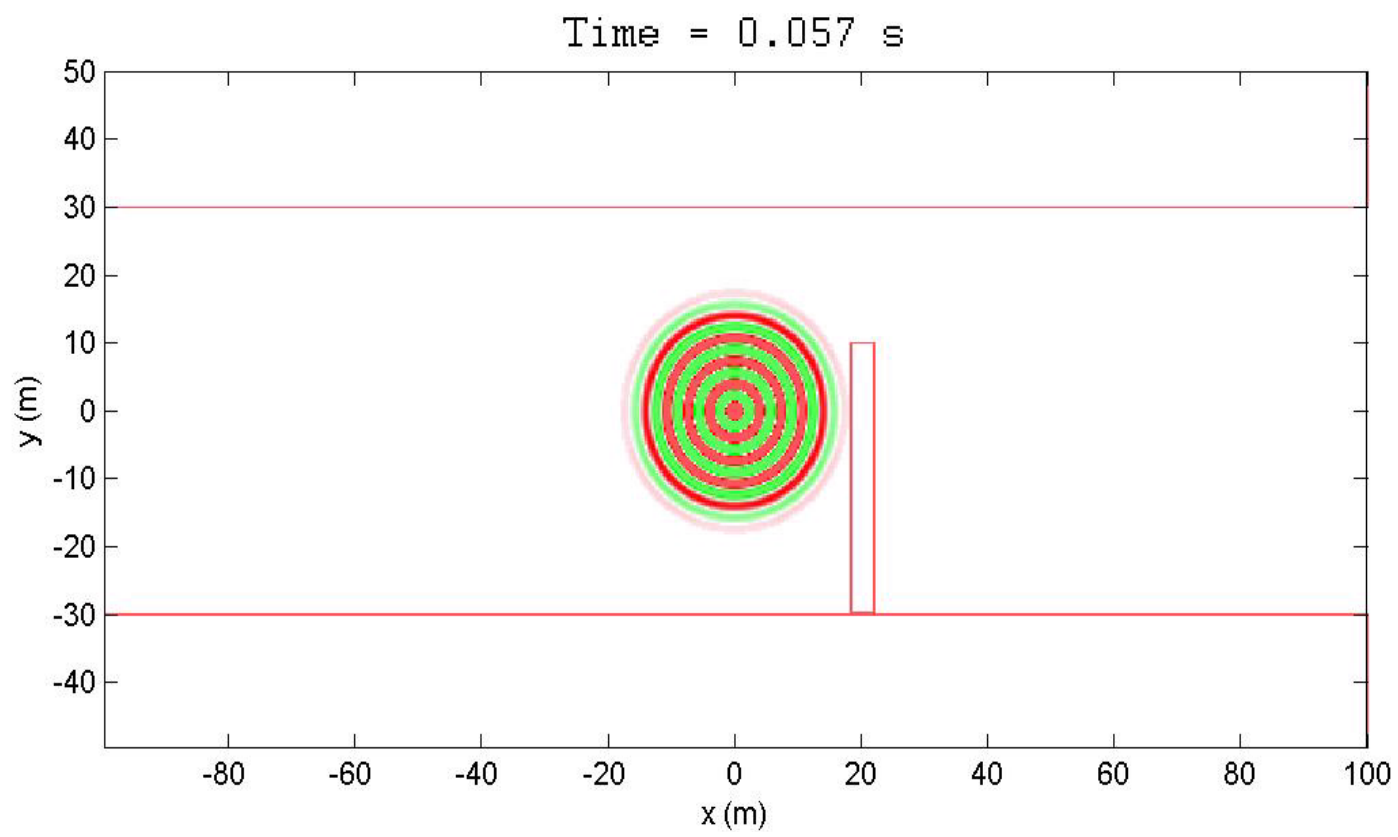

Figure 7. Propagation above soft, porous ground in the presence of a rigid barrier, $0.057 \mathrm{~s}$ after source initiation.

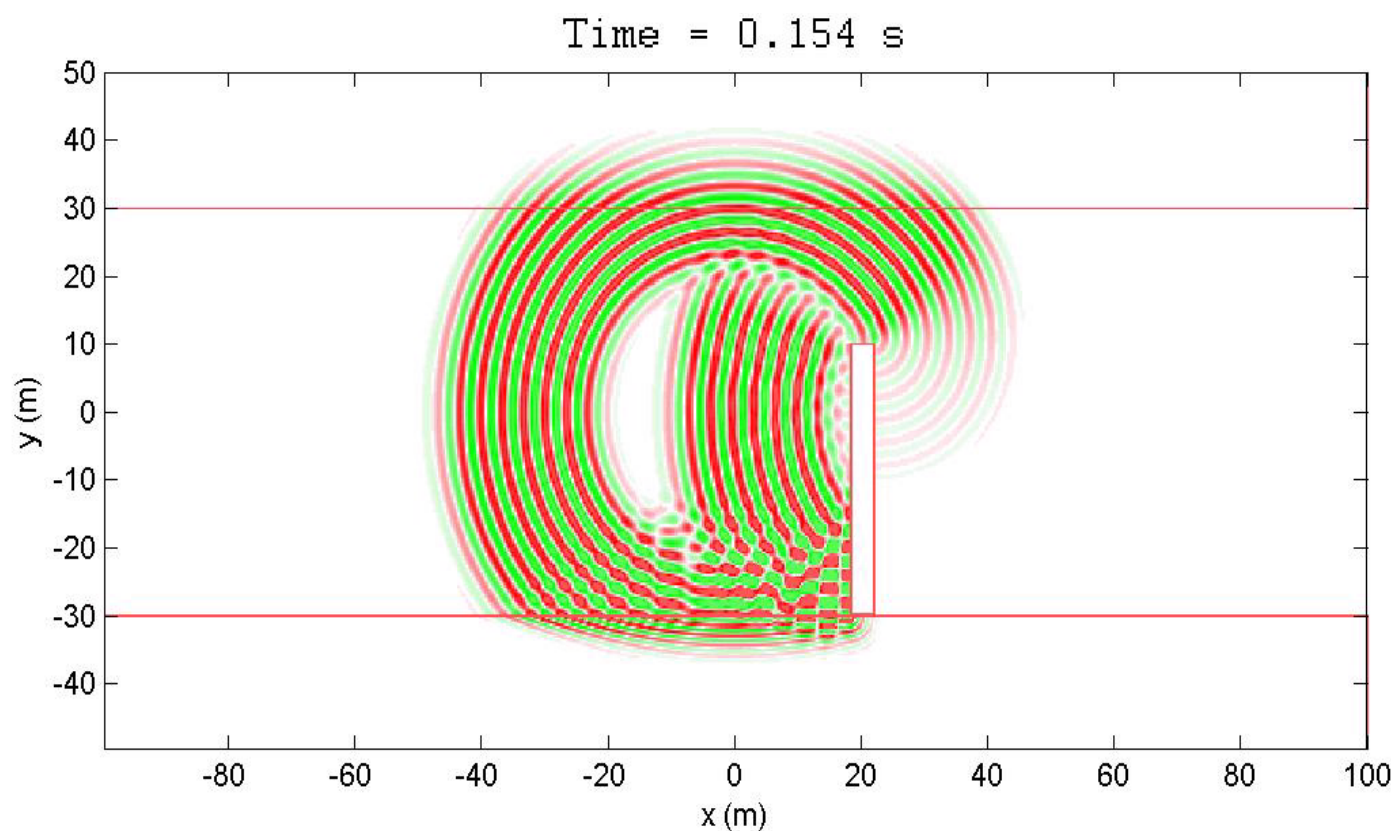

Figure 8. Propagation above soft, porous ground in the presence of a rigid barrier, $0.154 \mathrm{~s}$ after source initiation. 


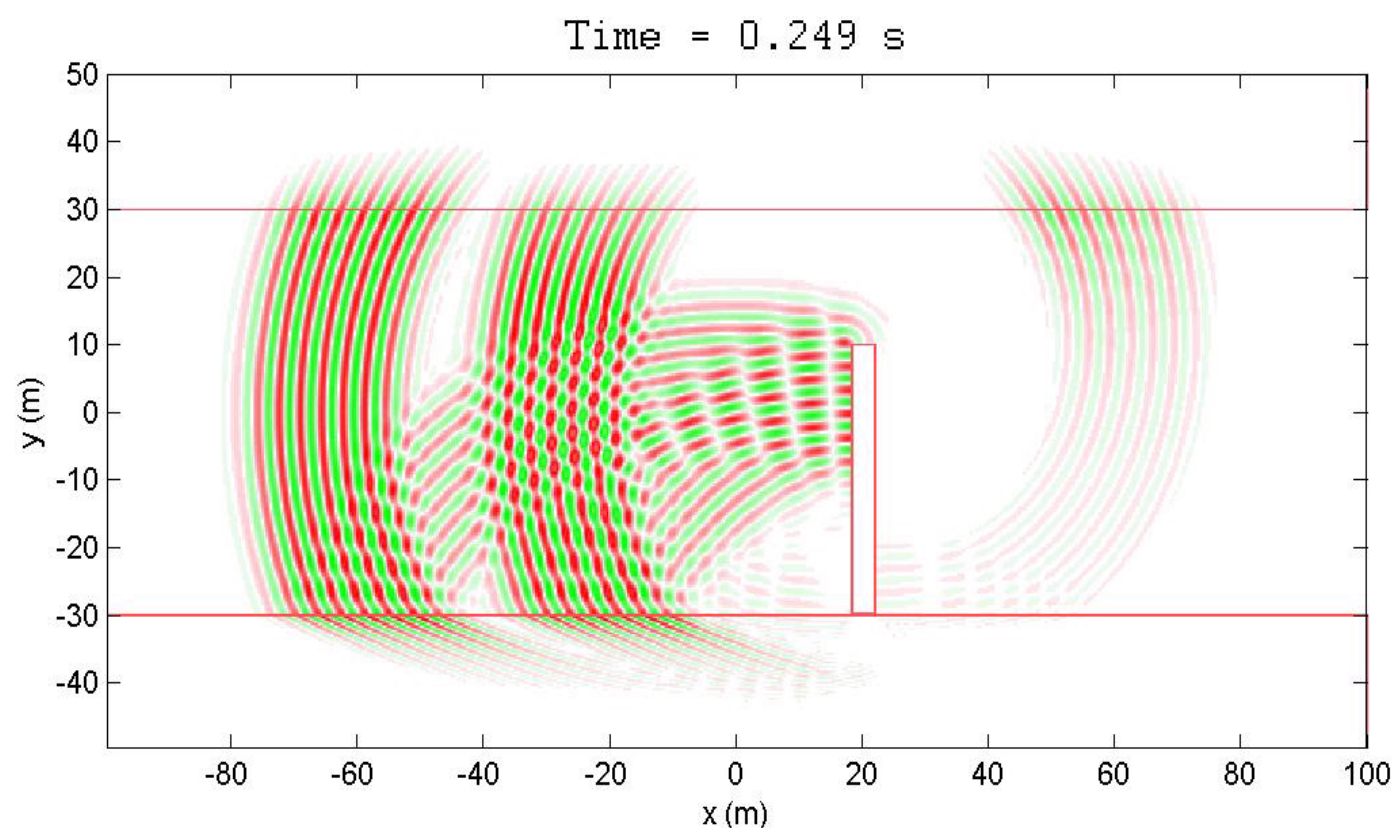

Figure 9. Propagation above soft, porous ground in the presence of a rigid barrier, $0.249 \mathrm{~s}$ after source initiation.

By comparison, a calculation for a moderately hard ground surface is shown in Figure 10. This calculation is identical to the preceding one, except that $\sigma$ has been increased to $10^{-4} \mathrm{~Pa}-\mathrm{s} \mathrm{m}^{-2}$. This value is characteristic of very soft soil. The pressure field $0.250 \mathrm{~s}$ after source initiation is shown. Notice that the higher value for the static flow resistivity causes the sound waves to reflect nearly perfectly from the ground in this case. 


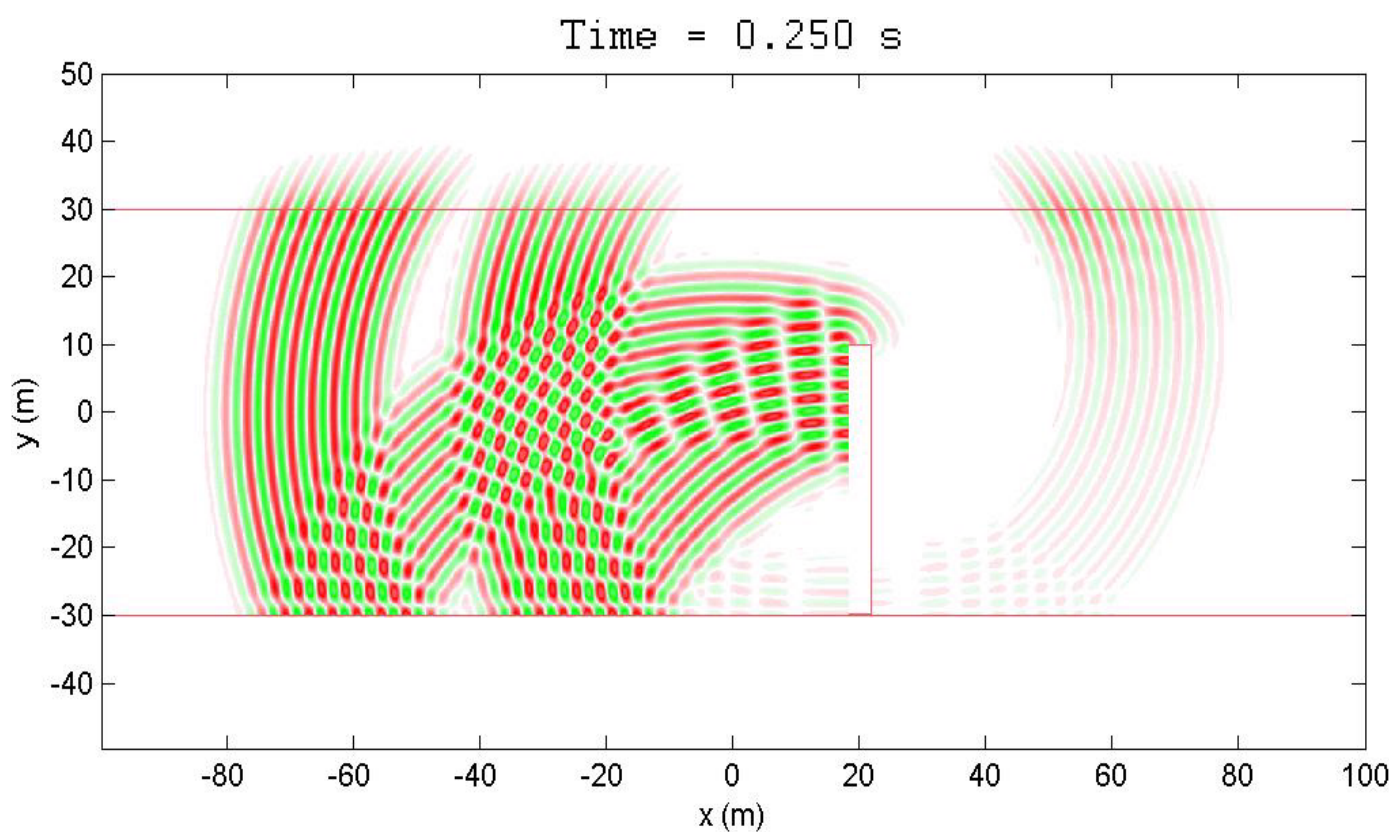

Figure 10. Propagation above moderately hard, porous ground in the presence of a rigid barrier, $0.250 \mathrm{~s}$ after source initiation.

Calculations involving porous media with flow resistivities exceeding $10^{-4}$ were also attempted but became unstable when the sound wave impinged on the porous layer. Since the porous layer is highly attenuative for large flow resistivities, the instability could have been caused by poor resolution of the spatial scales over which the wave attenuates.

Further research will be necessary to better understand the grid spacings and time steps necessary to achieve stability in a highly dissipative porous medium.

\section{High Mach Number, Uniform Flow}

In this section, calculations are considered for very-high-speed uniform flows. Although the flow speeds are much higher than would normally be encountered in the atmosphere, the calculations are very useful for testing the finite-difference implementation of the terms in Eq. 1 and 2 that are particular to a moving medium. The source is the same tapered, 10-cycle, $100-\mathrm{Hz}$ signal considered in the previous section. The domain size for all of the calculations is $100 \times 100 \mathrm{~m}$. Most of the calculations have 800 grid points in each direction, although a higher-resolution calculation with 1200 grid points is also discussed. Calculations at three Mach numbers are considered: 0, 0.3 (about 104 $\mathrm{m} / \mathrm{s}$ ), and 0.6 (about $207 \mathrm{~m} / \mathrm{s}$ ). The number of grid points per wavelength, in the upwind direction, is 27.2, 19.0, and 10.9, respectively, for these three Mach numbers. The fourthorder Runge-Kutta scheme with a Courant number of 0.4 was used to advance the wavefields in time. 
Figures 11-13 show the full, 2-D pressure fields at Mach 0, 0.3, and 0.6 after $0.11 \mathrm{~s}$ $(0.01 \mathrm{~s}$ after the source has terminated). Elongation of the waves propagating in the direction of the flow, and compression of the waves propagating counter to the flow, are clearly observed. (In the Mach 0.6 calculation, wavefronts appear on the left edge of the figure. These are wrapped around from the right edge by the periodic $\mathrm{BC}$.)

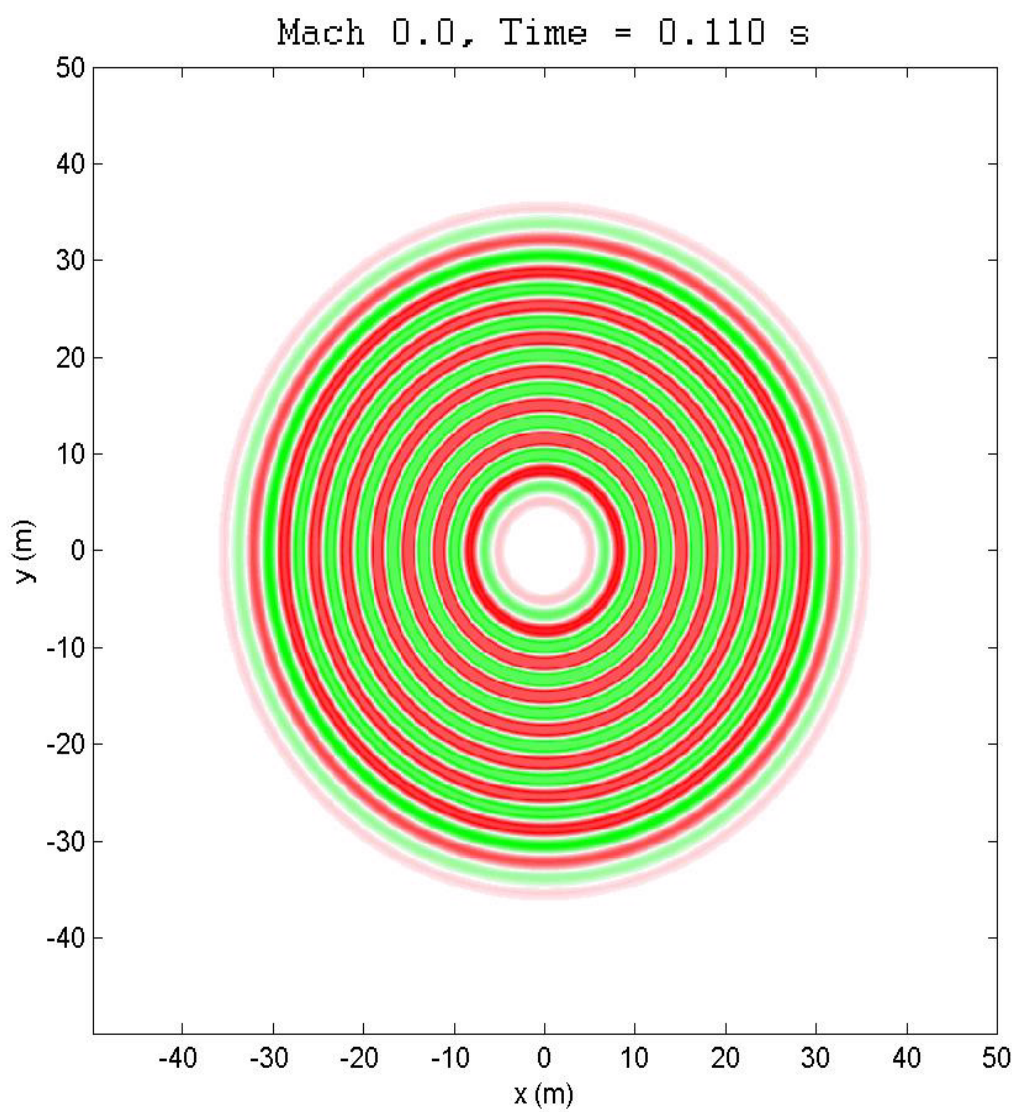

Figure 11. Sound pressure field in the absence of a background flow, Mach 0 flow. 


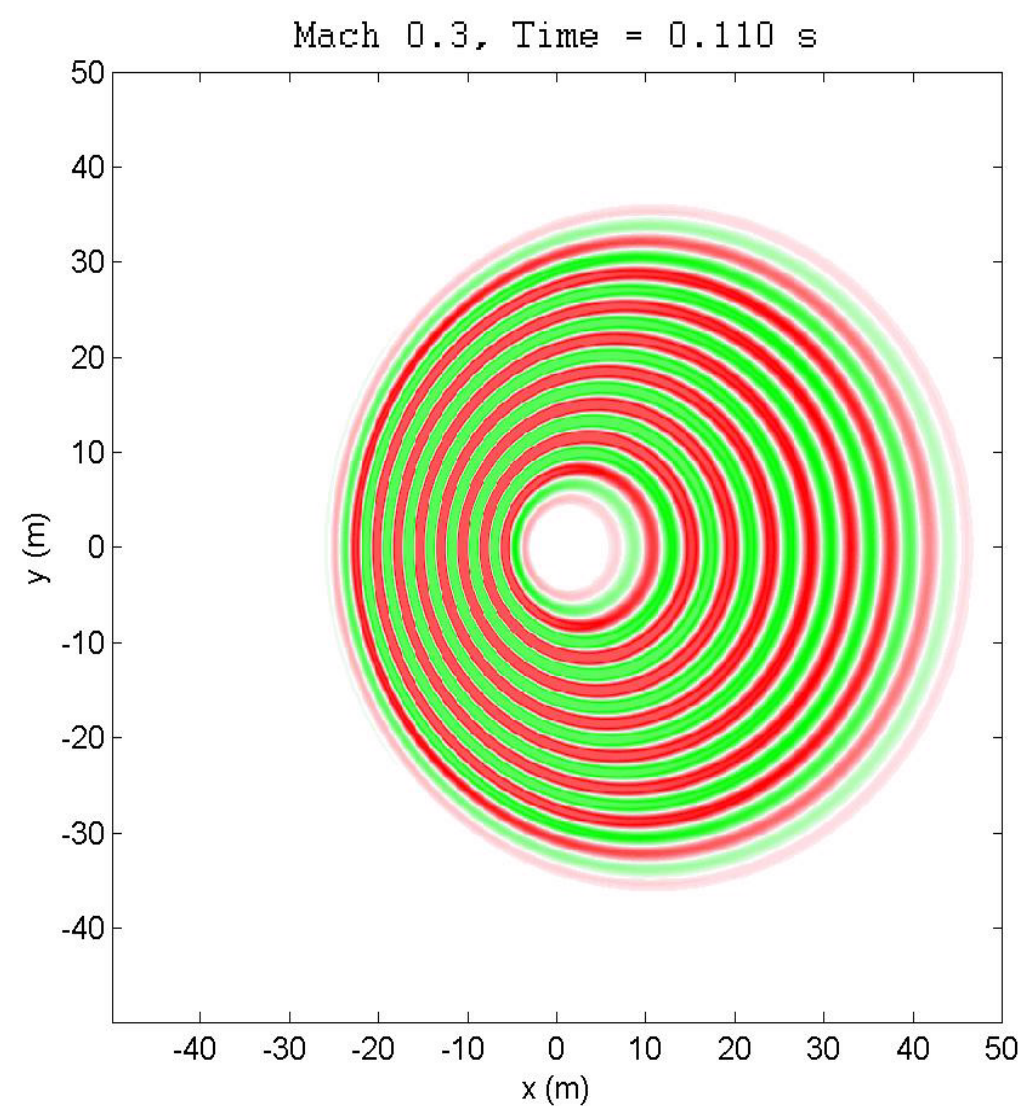

Figure 12. Sound pressure field for propagation in a uniform, Mach 0.3 flow. The flow direction is left to right. 


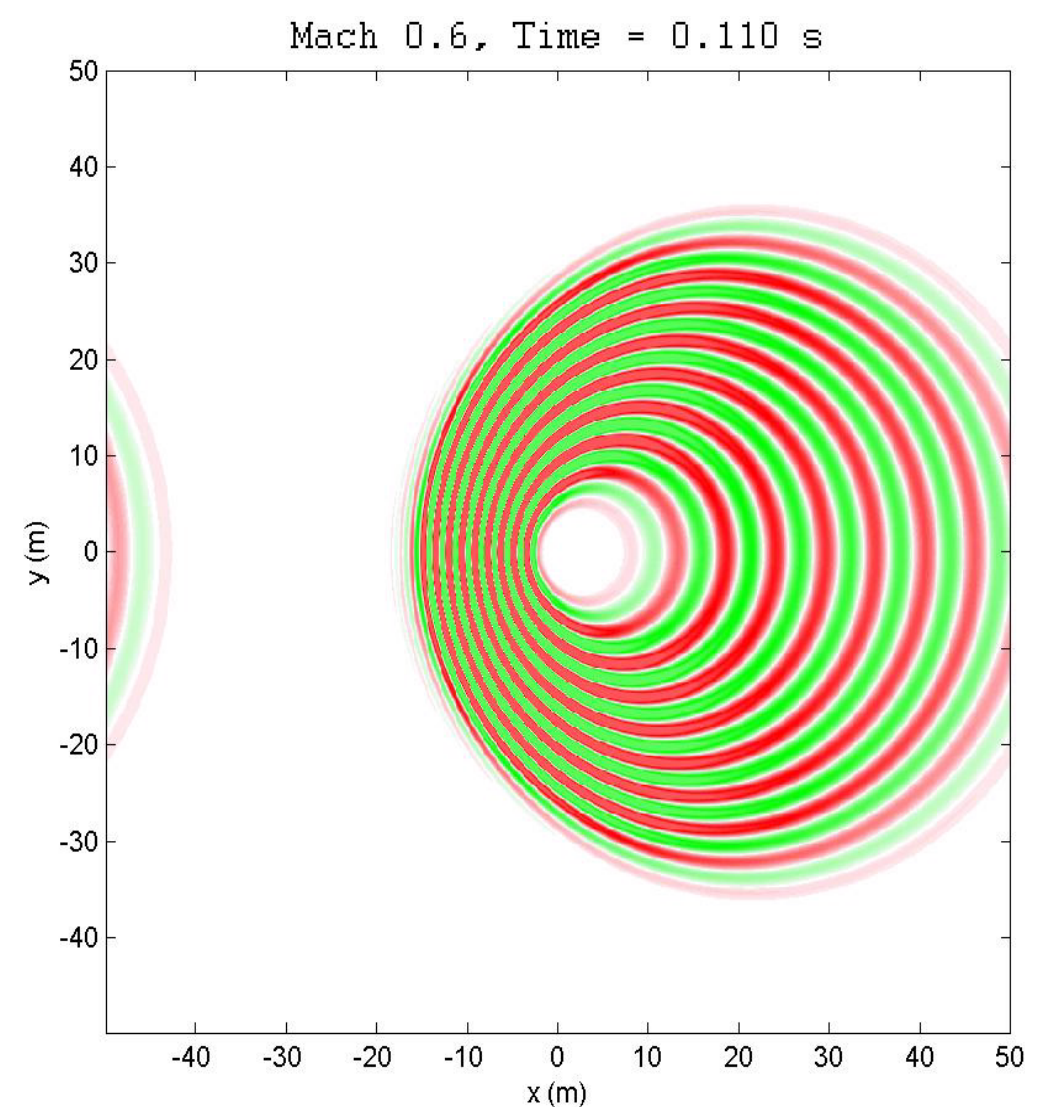

Figure 13. Sound pressure field for propagation in a uniform, Mach 0.6 flow. The flow direction is left to right.

Exact, time-domain solutions for 2-D wave propagation in a moving medium are not generally available. Recently, V.E. Ostashev ${ }^{*}$ has calculated the dependence of the pressure amplitude on azimuth for a monofrequency sound source radiating in two dimensions. The result is

$$
\frac{p(r, \theta, M)}{p(r, \theta, M=0)}=\frac{\left(1-M^{2} \sin ^{2} \theta\right)^{5}-M \cos \theta}{\left(1-M^{2} \sin ^{2} \theta\right)^{3 / 4}(1-M)}
$$

where $p(r, \theta, M)$ is the pressure amplitude as a function of cylindrical distance $r$, azimuthal angle $\theta$, and Mach number. This equation is intended for application in the farfield, that is, many wavelengths from the source. Figure 14 compares the FDTD calculations in the previous three figures to Ostashev's theory. Very close agreement is

*Personal communication, 2003. 
obtained at Mach 0.3. At Mach 0.6 the amplitude from the FDTD calculations is somewhat lower than expected in the upwind direction. A higher-resolution grid noticeably improves the predictions. Taken together, these results demonstrate that excellent agreement with Ostashev's theory can be obtained, although a very-high-resolution grid is required for high Mach numbers.

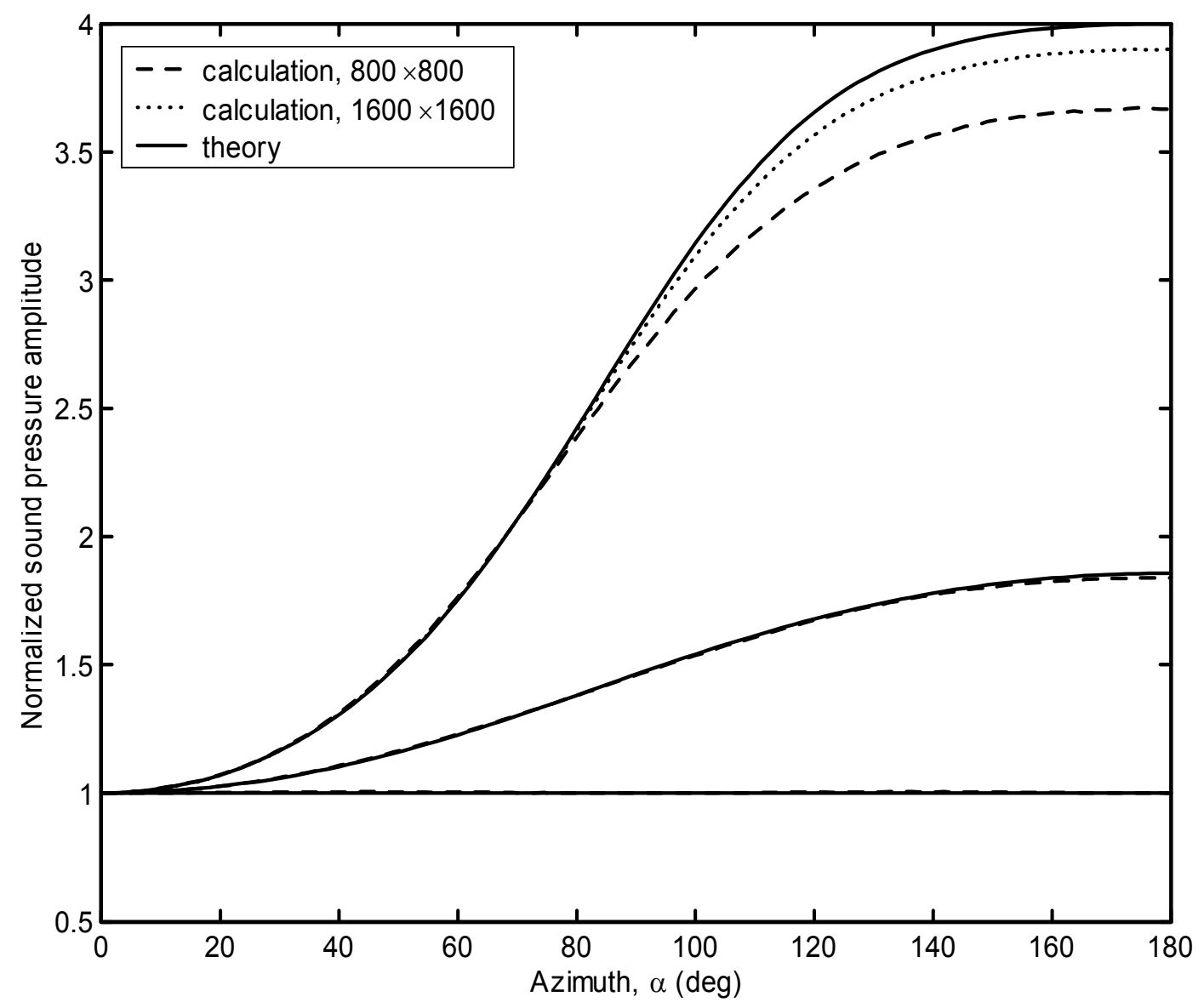

Figure 14. Comparison of the angular dependence of the pressure amplitude from the FDTD calculations of Ostashev's theory. The upwind direction is $0^{\circ}$ and the downwind direction is $180^{\circ}$.

Figures 15 and 16 repeat the Mach 0.3 calculation shown in Figure 12 except that different temporal integration methods are used. Results for the "staggered leap-frog" method (Fig. 15) are qualitatively similar to the fourth-order Runge-Kutta results. The time step used for the staggered leap-frog calculations was $1 / 4$ (Courant number $C=0.1$ ) that used for the fourth-order Runge-Kutta calculations, approximately equalizing the amount of computational effort involved (Table 2). Close examination of the near-source 
wavefronts propagating in the downwind direction reveals a noisy appearance not present in Figure 12. This noise is actually a growing numerical instability in the calculations. Such numerical instability is even more pronounced in the second-order Runge-Kutta calculations (Fig. 16). These calculations were performed with $C=0.2$.

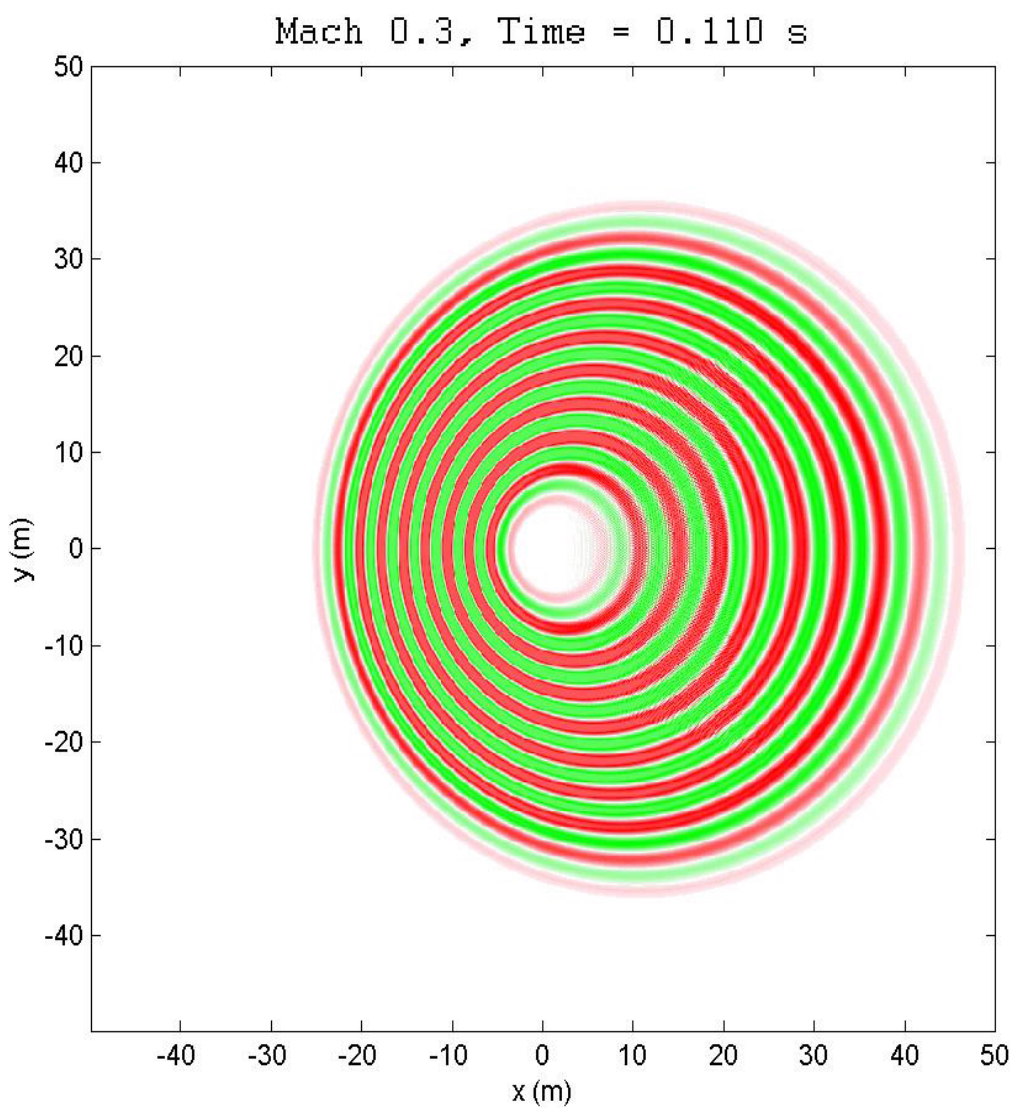

Figure 15. Sound pressure field for propagation in a uniform, Mach 0.3 flow. The staggered leap-frog method was used to advance the solution in time. 


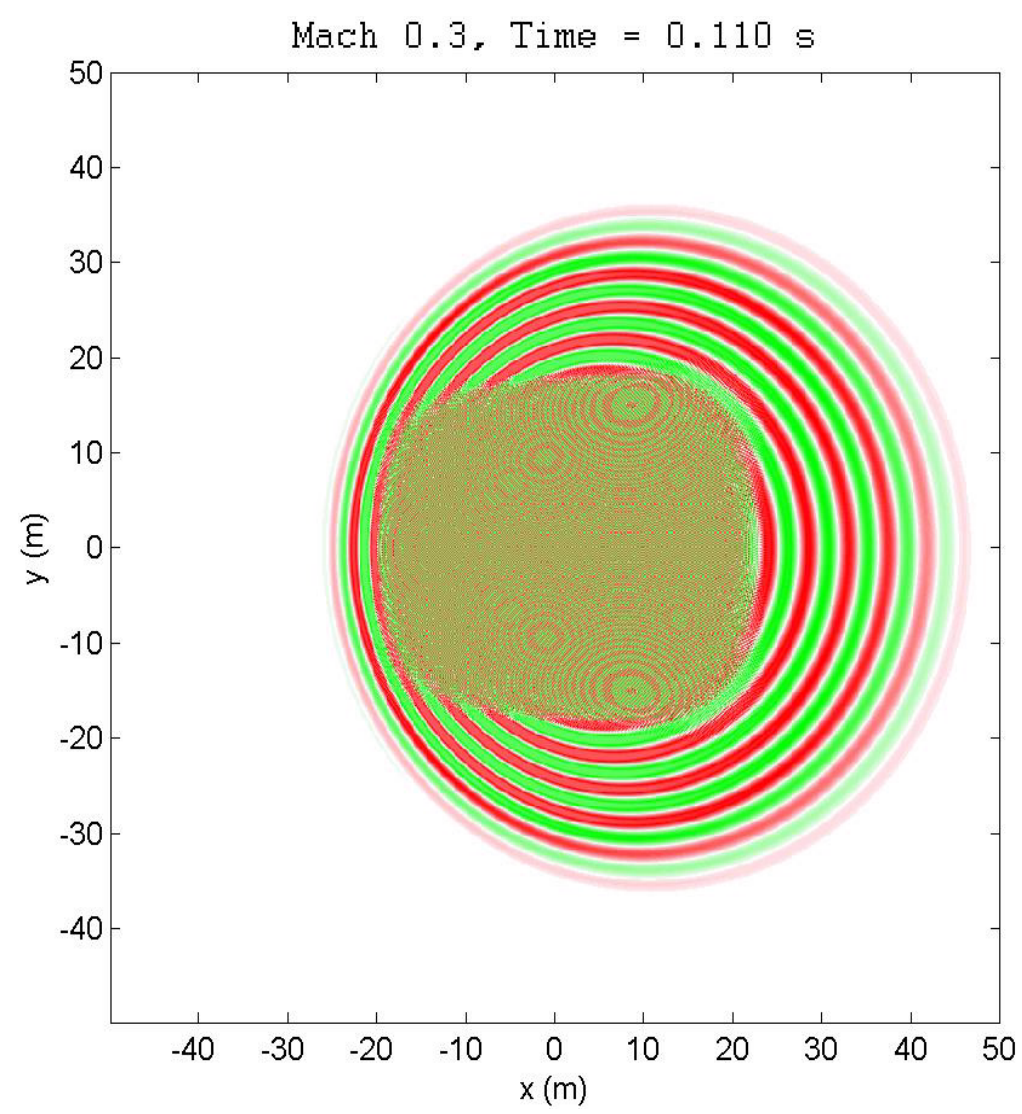

Figure 16. Sound pressure field for propagation in a uniform, Mach 0.3 flow. The second-order Runge-Kutta method was used to advance the solution in time.

Downwind and upwind results at Mach 0.3 are compared for several temporal integration methods in Figures 17 and 18. Shown are fourth-order Runge-Kutta $(C=0.4)$, unstaggered leap-frog $(C=0.1)$, and staggered leap-frog $(C=0.1)$. The first two of these methods yield graphically indistinguishable results. The staggered leap-frog scheme, however, yields a signal with a noticeably larger amplitude. The numerical instabilities are also clearly evident at small distances from the source. The lack of agreement between the staggered leap-frog scheme and the other methods, combined with its nonrigorous method of derivation, strongly suggests that it is inferior for this problem. 
Downwind

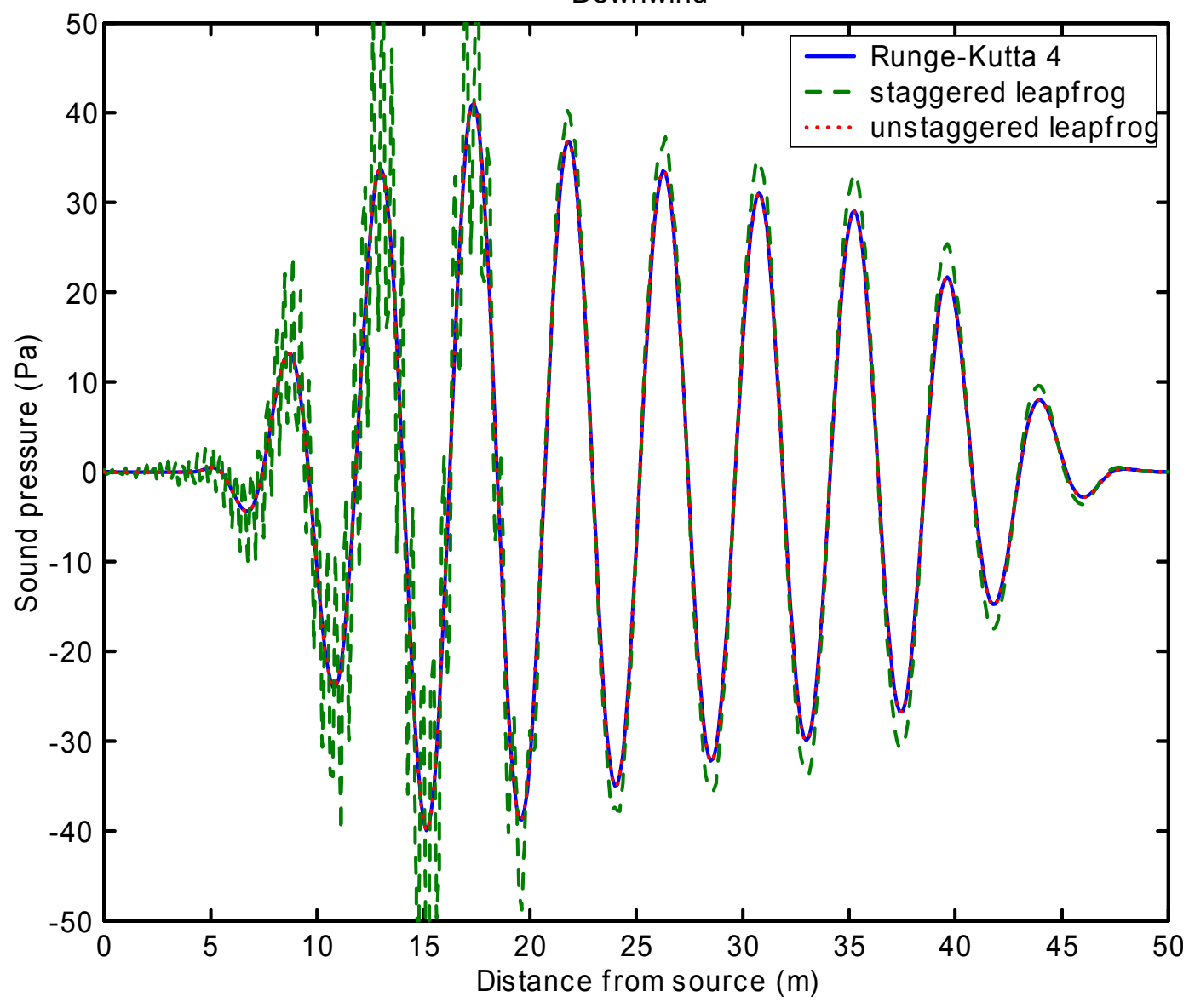

Figure 17. Sound pressure traces for downwind propagation at Mach 0.3. Shown are results for the fourth-order Runge-Kutta, staggered leap-frog, and unstaggered leap-frog methods. 


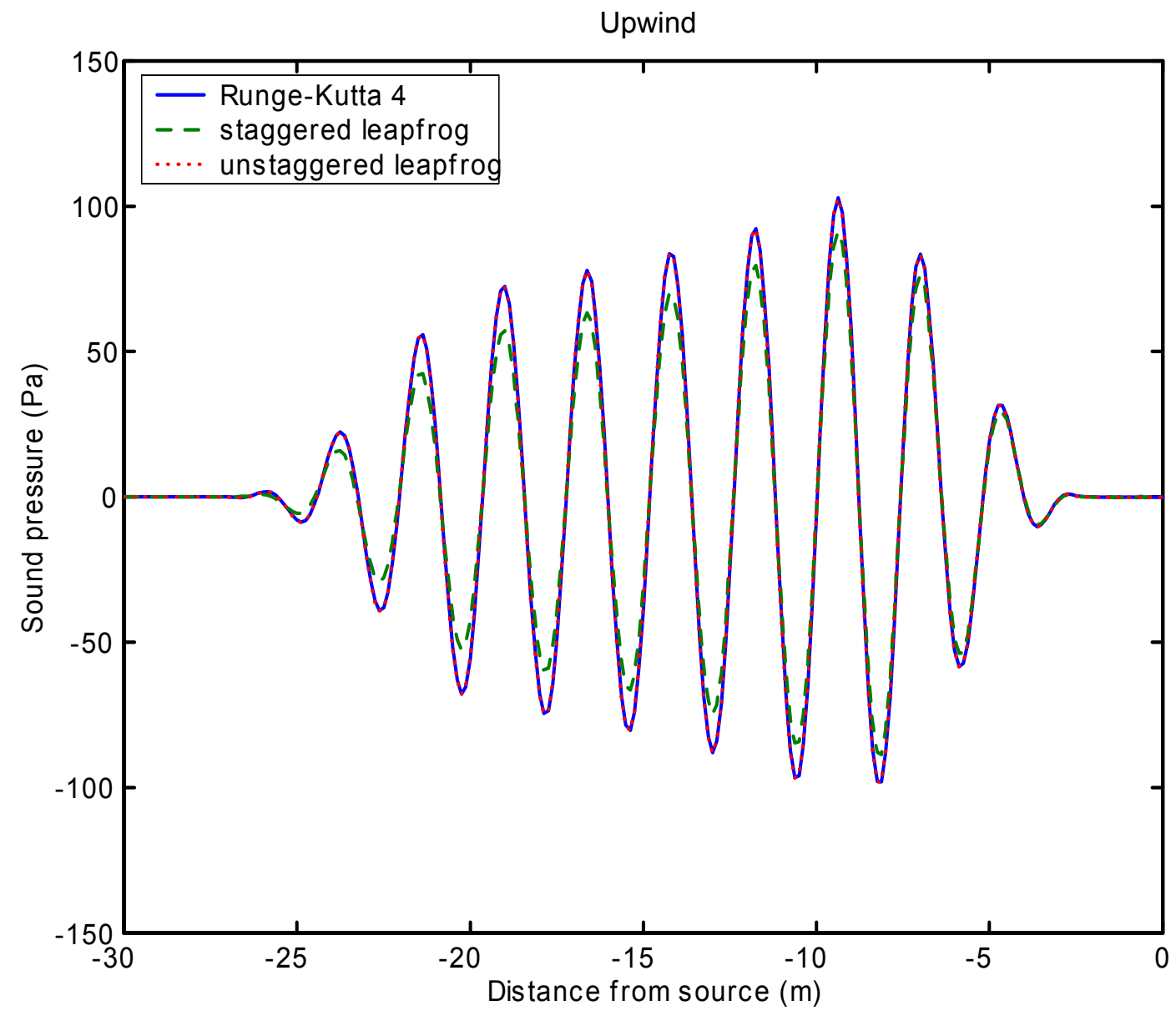

Figure 18. Sound pressure traces for upwind propagation at Mach 0.3 . Shown are results for the fourth-order Runge-Kutta, staggered leap-frog, and unstaggered leap-frog methods.

A similar comparison of downwind and upwind results, except at Mach 0.6, is shown in Figures 19 and 20. The temporal integration methods shown in these figures are fourthorder Runge-Kutta $(C=0.4)$, unstaggered leap-frog $(C=0.1)$, and Aldridge $(C=0.1)$. The second-order Runge-Kutta and staggered leap-frog methods were both extremely unstable at this Mach number and therefore are not shown. The three methods displayed in the figure, however, were all stable and provide graphically indistinguishable results. 


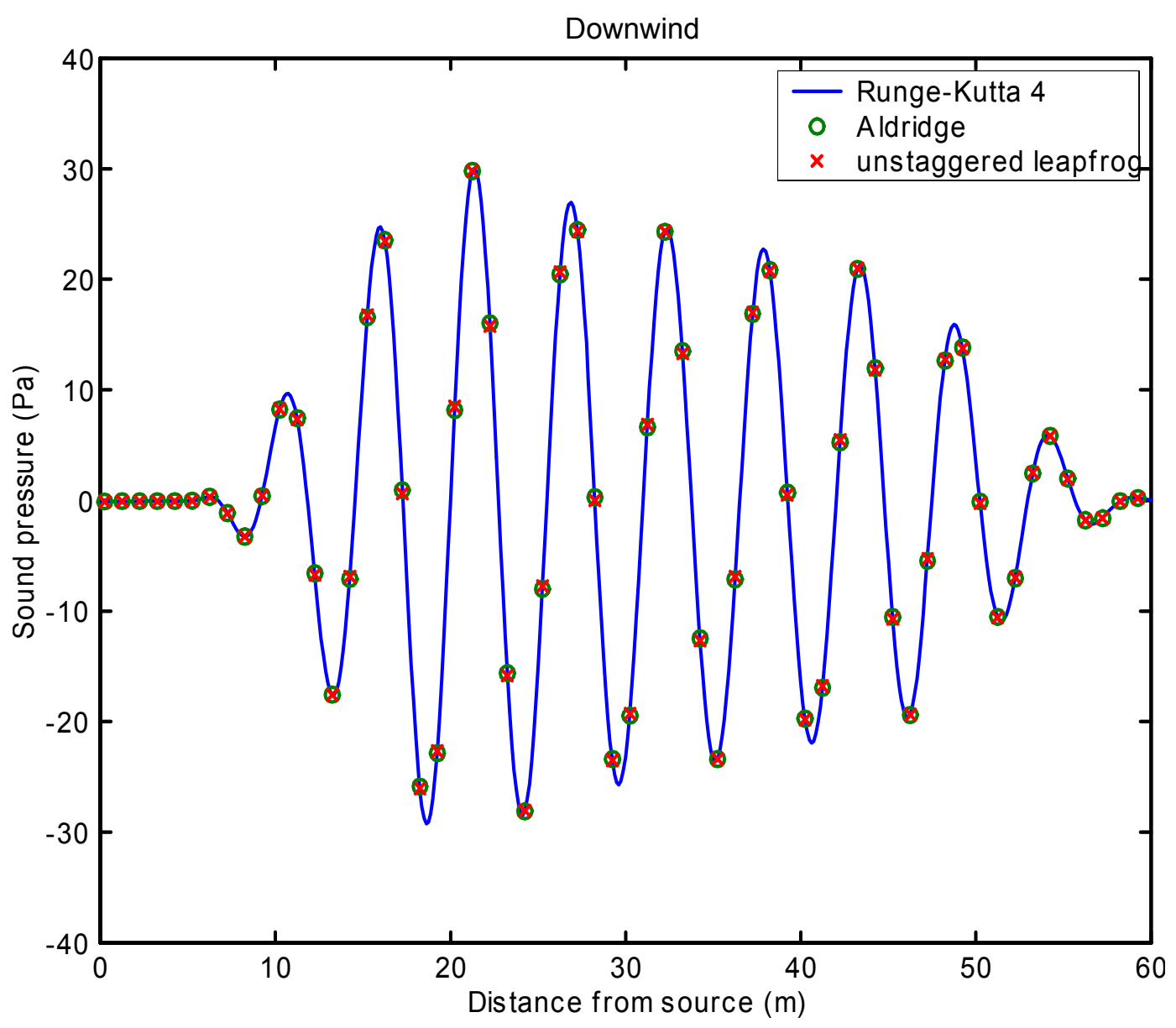

Figure 19. Sound pressure traces for downwind propagation at Mach 0.6. Shown are results for the fourth-order Runge-Kutta, Aldridge, and unstaggered leap-frog methods. 
Upwind

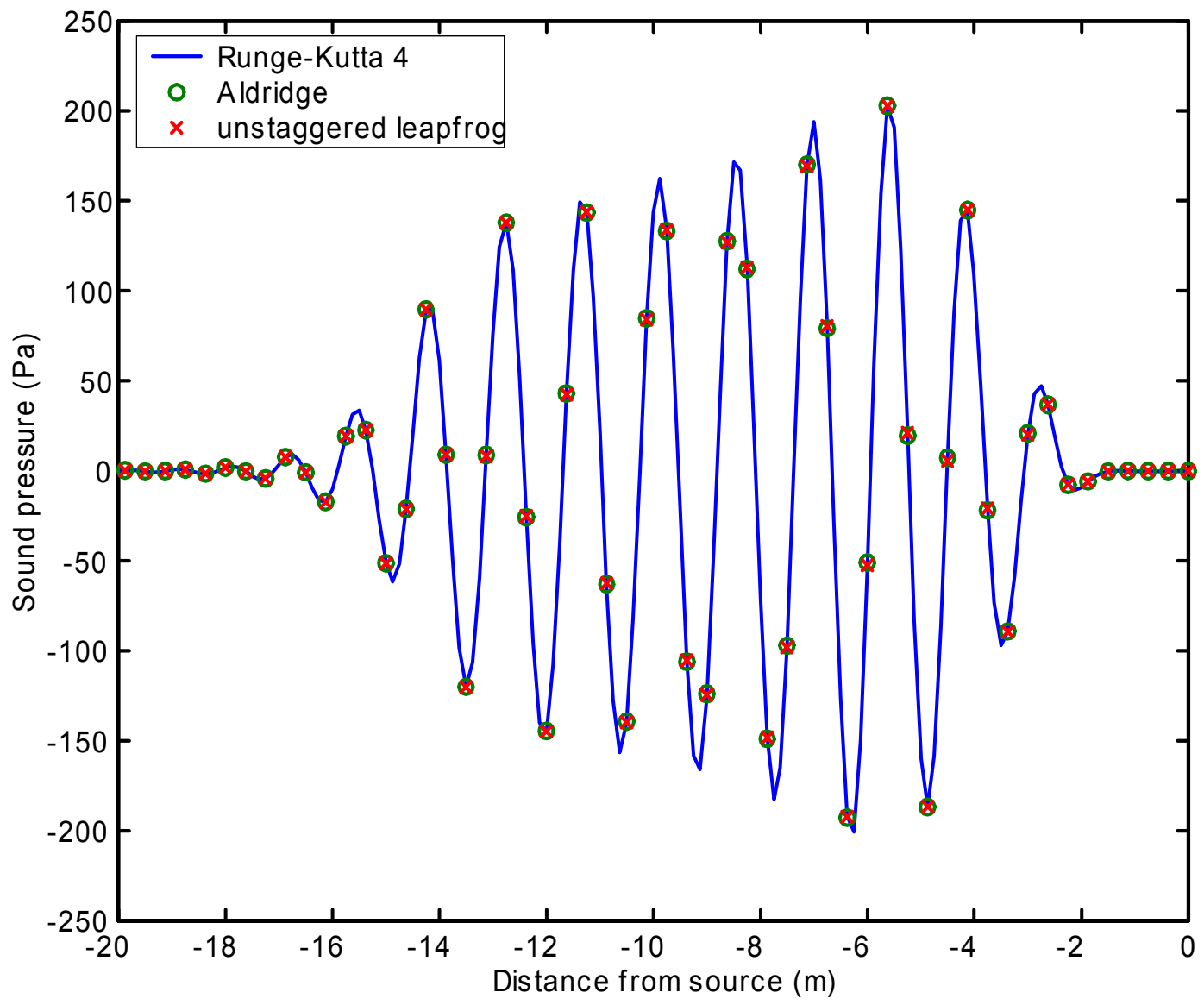

Figure 20. Sound pressure traces for upwind propagation at Mach 0.6. Shown are results for the fourth-order Runge-Kutta, Aldridge, and unstaggered leap-frog methods.

Based on these comparisons of the temporal integration methods, it appears that the unstaggered leap-frog, Aldridge, and fourth-order Runge-Kutta methods all provide good accuracy and stability for a similar level of computational effort. More detailed analysis would be necessary to determine which of these methods is most satisfactory. From a practical standpoint, any of them appears to be a reasonable choice.

\section{Nonuniform (Turbulent) Flow}

Atmospheric turbulence has several important effects on signals received by acoustic sensors: it causes signal levels to fluctuate randomly, it distorts wavefronts (thereby changing angles-of-arrival and hindering source direction finding), and it scatters sound energy over and around objects. Therefore, incorporation of turbulence into FDTD 
calculations is of much practical importance. Two general approaches to generating the necessary turbulence input fields are possible: kinematic and dynamic. The kinematic method consists of synthesizing random fields that possess certain desired statistical properties characteristic of actual turbulence. The dynamic method consists of simulating actual turbulence with fluid dynamical equations.

The primary advantages of the kinematic methods are low computational burden, the ability to readily generate fields at a high spatial resolution, and tight control over statistical properties. This last feature is useful, for example, in comparing the FDTD calculations to known analytical solutions. The primary advantage of the dynamic methods is that the temporal properties and the higher-order statistics of the fields are as close as possible to the actual atmosphere. The main dynamic method of interest for FDTD calculations at Army tactical scales is large-eddy simulation, or LES (Moeng 1998). At present, LES is capable of providing inputs to an FDTD code at a resolution of several meters (e.g., Sullivan et al. 1996). Conceivably the LES could be directly coupled with the acoustical calculation by simultaneously solving a single set of fluid equations. However, such a procedure can lead to poor results because of the discrepancy between the dominant scales of the atmospheric turbulence process and the acoustic wavelength, as well as the small magnitude of the acoustic fluctuations relative to the atmospheric ones.

For illustrative purposes we consider here a single example of FDTD calculation of sound propagation through turbulence. The example uses the recently developed technique for generating kinematic turbulence called quasi-wavelets (QWs). Like ordinary wavelets, QWs are based on rescaling and translation of a specified parent function. Unlike ordinary wavelets, the positions and orientations of the QWs are random. Goedecke et al. (2003) derived a random QW distribution and parent function that exactly reproduce a prescribed turbulence spectrum such as von Kármán's. Figures 21 and 22 show snapshots of $v_{x}$ and $v_{y}$, respectively, for a random QW field. As is characteristic of atmospheric turbulence, there are strong but infrequent patches of large-scale velocity fluctuations, interspersed with more numerous but weaker small-scale patches. FDTD calculations of the propagation of a six-cycle burst at $100 \mathrm{~Hz}$ through the QW fields is shown in Figure 23. Note the increasing distortions to the wavefronts as they propagate from the source. This example is somewhat exaggerated in that the turbulent velocity fluctuations have a standard deviation of about $10 \mathrm{~m} \mathrm{~s}^{-1}$, which is an order of magnitude higher than would typically be encountered in practice. However, the propagation distance is much shorter than typical in Army tactical scenarios. 


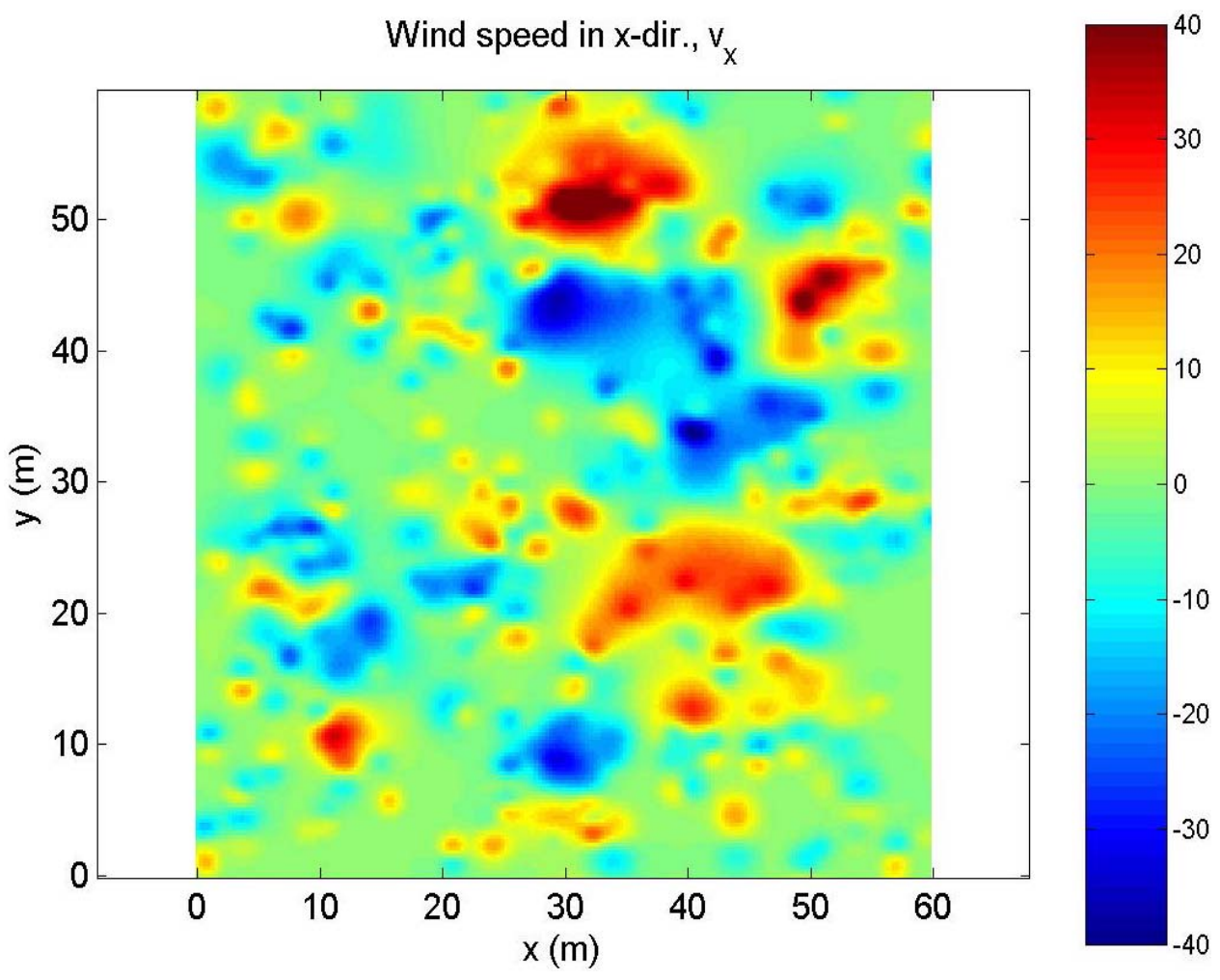

Figure 21. Snapshot of a random turbulence field generated by the quasiwavelet method. The $x$ component of the wind velocity is shown. 


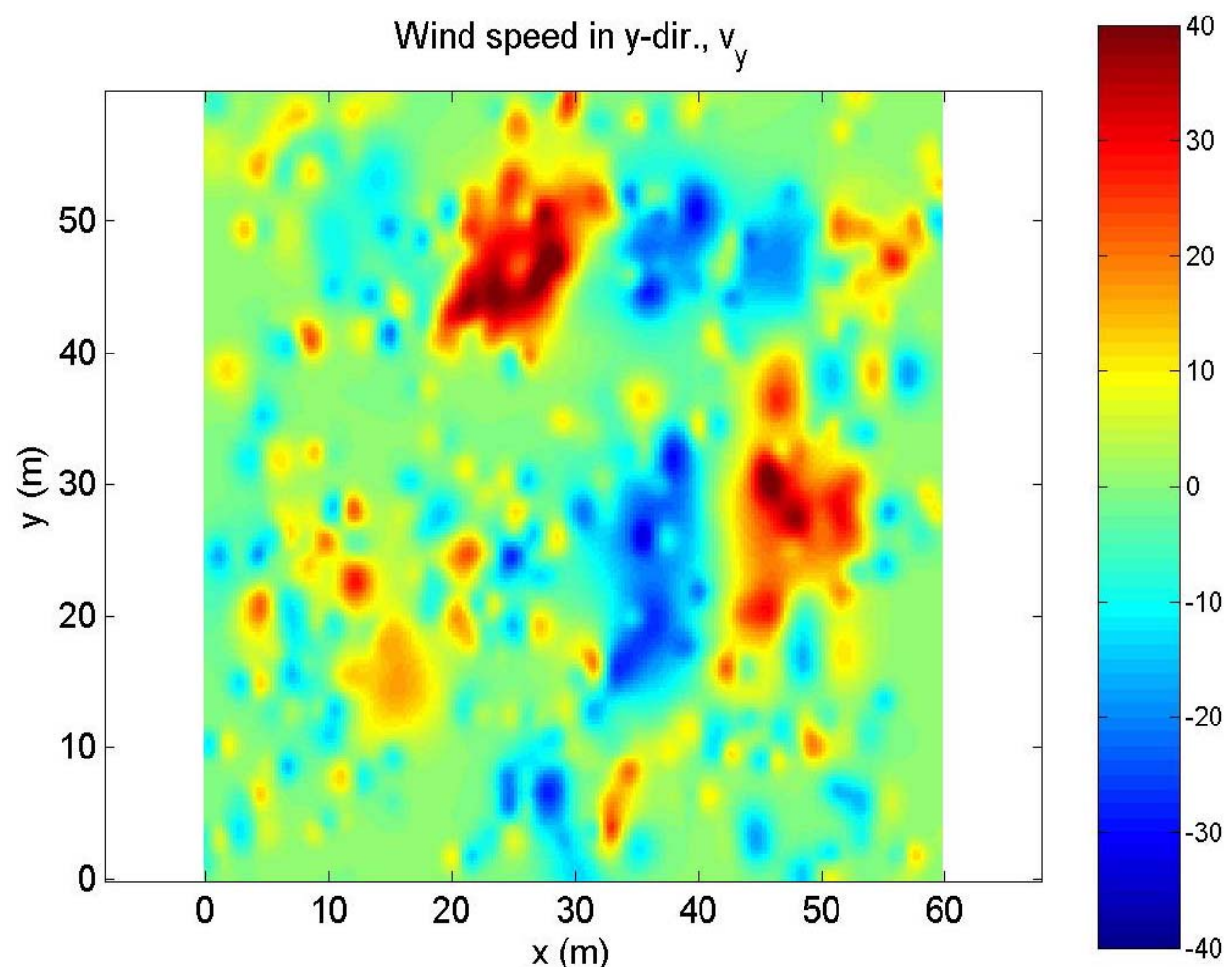

Figure 22. Same as Figure 21, except that the $y$ component of the wind velocity is shown. 


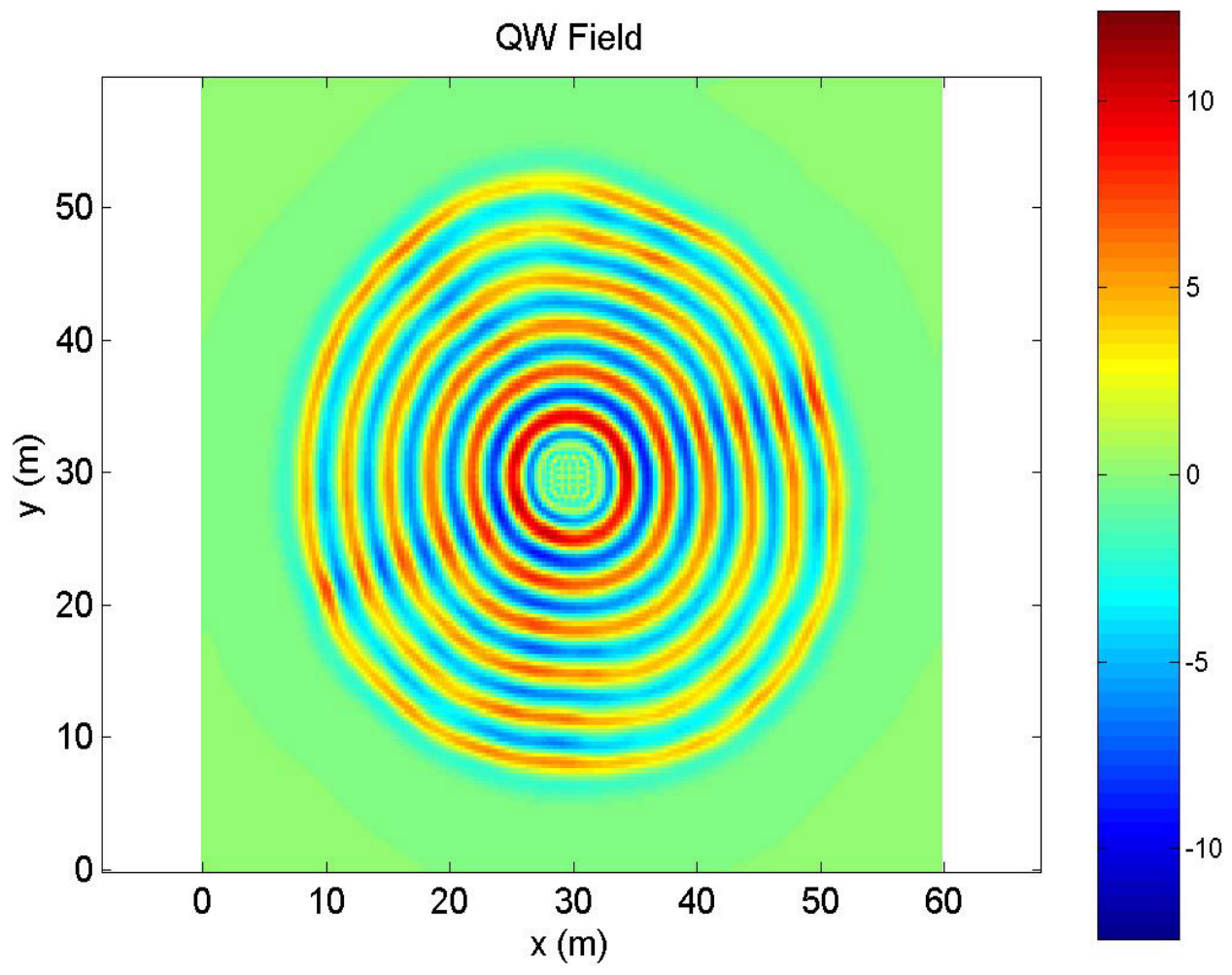

Figure 23. Propagation of a six-cycle burst at $100 \mathrm{~Hz}$ through the turbulence fields shown in Figures 21 and 22. 


\section{CONCLUSION}

This report has addressed many aspects of finite-difference, time-domain (FDTD) simulation of sound wave propagation in a moving medium, from the theoretical equations upon which it is based to practical issues of numerical implementation. One of the most important insights is that motion in the propagation medium makes impossible direct implementation of the customary, staggered grid schemes used for other types of wave propagation problems. This is because of the presence of the advective terms in the differential equations, which do not naturally center themselves on the grid points where they are needed. Therefore, alternative finite-difference formulations must be considered.

The numerical approaches considered in this report were all based on the customary, staggered spatial grid, in which the pressure and particle velocities are displaced by onehalf of the grid interval. Advancement of the wavefield variables in time was then considered in terms of unstaggered and staggered temporal grids. Two temporally unstaggered schemes - the fourth-order Runge-Kutta method and the unstaggered "leap-frog" method-were found to be quite satisfactory. A temporally staggered scheme, with fields stored from two previous time levels, also performed well. However, a non-rigorous, staggered, leap-frog scheme analogous to the one currently in widespread use for FDTD wave propagation calculations in a non-moving medium was found to be inaccurate. Additional comparisons of the various temporal integration schemes would be very worthwhile.

Rigid boundary conditions and porous ground layers were also considered in this report. The rigid condition is useful for modeling buildings, barriers, tree trunks, and other acoustically hard objects. A simple, low-frequency model for the porous ground can be easily incorporated into an acoustic FDTD code. However, the utility of this model is limited to materials with low flow resistivities, such as snow or coarse sand. Therefore, it would be highly desirable to develop other approaches to implementing the ground material, such as a ground-layer model with applicability to a broader frequency range or an impedance boundary condition that does not require an actual ground layer. 


\section{REFERENCES}

Aldridge, D.F. (2002) Acoustic wave equations for a linear viscous fluid and an ideal fluid. Technical Report SAND2002-2060, Sandia National Laboratories, Albuquerque, New Mexico. (Available online at http://www.ntis.gov/ordering. htm.)

Attenborough, K. (1983) Acoustical characteristics of rigid fibrous absorbents and granular materials. Journal of the Acoustical Society of America, 73: 785-799.

Attenborough, K., S. Taherzadeh, H.E. Bass, X. Di, R. Raspet, G.R. Becker, A. Güdesen, A. Chrestman, G.A. Daigle, A.L. Esprance, Y. Gabillet, K.E. Gilbert, Y.L. Li, J. White, P. Naz, J.M. Noble, and H.A.J.M. van Hoof (1995) Benchmark cases for outdoor sound propagation models. Journal of the Acoustical Society of America, 97: 173-191.

Berenger, J.P. (1994) A perfectly matched layer for the absorption of electromagnetic waves. Journal of Computational Physics, 114: 185-200.

Blumrich, R., and D. Heimann (2002) A linearized Eulerian sound propagation model for studies of complex meteorological effects. Journal of the Acoustical Society of America, 112: 446-455.

Botteldooren, D. (1994) Acoustical finite-difference time-domain simulation in a quasiartesian grid. Journal of the Acoustical Society of America, 95: 2313-2319.

Burden, R.L., J.D. Faires, and A.C. Reynolds (1981) Numerical Analysis. Second edition. Boston: Prindle, Weber, and Schmidt.

Cerjan, C., R. Kosloff, and M. Reshef (1985) A nonreflecting boundary condition for discrete acoustic-wave and elastic-wave equations. Geophysics, 50: 705-708.

Goedecke, G., V.E. Ostashev, D.K. Wilson, and H.J. Auvermann (2003) Quasiwavelet model of von Kármán and other velocity spectra. Boundary-Layer Meteorology, accepted.

Kasahara, A. (1977) Computational aspects of numerical models for weather prediction and climate simulation. In Methods in Computational Physics (J. Chang, ed.). New York: Academic Press, p. 1-66.

Kinsler, L.E., A.R. Frey, A.B. Coppens, and J.V. Sanders (1982) Fundamentals of Acoustics. New York: Wiley and Sons.

Kreyszig, E. (1988) Advanced Engineering Mathematics. New York: Wiley and Sons.

Lingevitch, J.F., M.D. Collins, D.K. Dacol, D.P. Drob, J.C.W. Rogers, and W.L. Siegmann (2002) A wide angle and high Mach number parabolic equation. Journal of the Acoustical Society of America, 111: 729-734. 
Liu, L., and M.L. Moran (2002) Acoustic wave propagation in atmospheric boundary layer above rough terrain. Journal of the Acoustical Society of America, 111: 2378-2379. Abstract only.

Moeng, C.-H. (1998) Large eddy simulation of atmospheric boundary layers. In Clear and Cloudy Boundary Layers. Amsterdam: Royal Netherlands Academy of Arts and Sciences, p. 66-83.

Morse, P.M. and K.U. Ingard (1968) Theoretical Acoustics. New York: McGraw-Hill.

Ostashev, V.E. (1987) On sound wave propagation in a three-dimensional inhomogeneous moving medium. In Russian. In Diffraction and Wave Propagation in Inhomogeneous Media. Moscow: MPTI Press, p. 42-49.

Ostashev, V.E. (1997) Acoustics in Moving Inhomogeneous Media. London: E and FN Spon.

Ostashev, V.E., D. Juvé, and P. Blanc-Benon (1997) Derivation of a wide-angle parabolic equation for sound waves in inhomogeneous moving media. Acustica, 83: 455-460.

Pierce, A.D. (1990) Wave equation for sound in fluids with unsteady inhomogeneous flow. Journal of the Acoustical Society of America, 87: 2292-2299.

Salomons, E.M. (2001) Computational Atmospheric Acoustics. Dordrecht: Kluwer.

Salomons, E.M., R. Blumrich, and D. Heimann (2002) Eulerian time-domain model for sound propagation over a finite-impedance ground surface. Comparison with frequency-domain models. Acustica/Acta Acustica, 88: 483-492.

Sullivan, P.P., J.C. McWilliams, and C.-H. Moeng (1996) A grid nesting method for large-eddy simulation of planetary boundary-layer flows. Boundary-Layer Meteorology, 80: $167-202$.

Yee, K.S. (1966) Numerical solution of initial boundary value problems involving Maxwell's equations in isotropic media. IEEE Trans., Antennas and Propagation, 14: 302307.

Yuan, X., D. Borup, J. Wiskin, M. Berggren, R. Eidens, and S. Johnson (1997) Formulation and validation of Berenger's PML absorbing boundary for the FDTD simulation of acoustic scattering. IEEE Trans., Ultrasonic Perroelectrics and Frequency Control, 44: 816-822. 


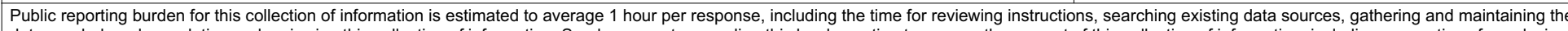

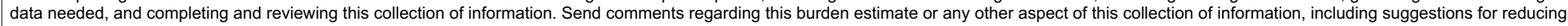

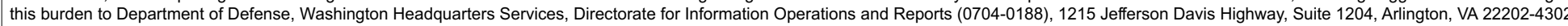

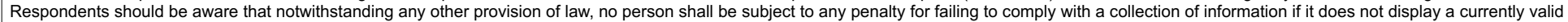
OMB control number. PLEASE DO NOT RETURN YOUR FORM TO THE ABOVE ADDRESS.
1. REPORT DATE (DD-MM-YY)
2. REPORT TYPE
May 2004
Technical Report
3. DATES COVERED (From - To)

4. TITLE AND SUBTITLE

5a. CONTRACT NUMBER

Finite-Difference, Time-Domain Simulation of Sound Propagation in a Dynamic Atmosphere

5b. GRANT NUMBER

5c. PROGRAM ELEMENT NUMBER

\section{AUTHOR(S)}

D. Keith Wilson and Lanbo Liu

5d. PROJECT NUMBER

5e. TASK NUMBER

5f. WORK UNIT NUMBER

8. PERFORMING ORGANIZATION REPORT

7. PERFORMING ORGANIZATION NAME(S) AND ADDRESS(ES)

U.S. Army Engineer Research and Development Center

Cold Regions Research and Engineering Laboratory

72 Lyme Road

ERDC/CRREL TR-04-12

Hanover, NH 03755-1290

9. SPONSORING/MONITORING AGENCY NAME(S) AND ADDRESS(ES)

10. SPONSOR / MONITOR'S ACRONYM(S)

U.S. Army Corps of Engineers

Washington, DC 20314-1000

11. SPONSOR / MONITOR'S REPORT NUMBER(S)

\section{DISTRIBUTION / AVAILABILITY STATEMENT}

Approved for public release; distribution is unlimited.

Available from NTIS, Springfield, Virginia 22161.

\section{SUPPLEMENTARY NOTES}

\section{ABSTRACT}

Finite-difference, time-domain (FDTD) techniques hold much promise for performing realistic simulations of sound propagation through complex, dynamic outdoor environments. This report focuses on a key aspect of FDTD in the atmosphere, namely the incorporation of a moving background medium (wind and turbulence in the atmosphere) into the calculations. Appropriate differential equations for FDTD simulation of sound propagation in a moving fluid are discussed. It is shown that FDTD calculations are not possible with this equation set when using the staggered grid, "leap-frog" approach, which is common for FDTD simulation of other types of wave propagation. Various finite-difference operators that are valid for a moving medium, such as Runge-Kutta and an unstaggered leap-frog approach, are discussed and compared. It is shown that a rigorous FDTD solution in a moving medium requires storing the field variables over at least two time steps, thereby requiring at least twice as much computer memory as the customary staggered grid. Several other topics pertinent to FDTD simulation of sound propagation in the atmosphere are discussed, including implementation of porous ground layers, absorbing boundaries, and rigid surfaces. Example calculations demonstrate the performance of the various finite-difference operators for a high Mach number, uniform flow. Other example calculations show FDTD calculations for propagation above rigid and porous ground surfaces, over rigid barriers, and through turbulence. With sufficiently dense spatial grids, very good agreement can be obtained between the FDTD calculations and known theoretical solutions.

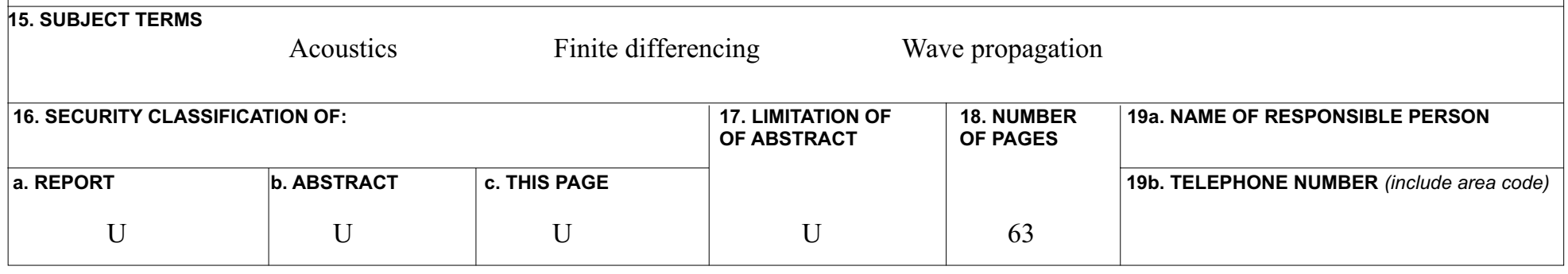

